\title{
Surface wave scattering by multiple flexible fishing cage system
}

Cite as: Phys. Fluids 33, 037119 (2021); https://doi.org/10.1063/5.0040662

Submitted: 15 December 2020 • Accepted: 20 February 2021 • Published Online: 19 March 2021

Siluvai Antony Selvan, (D) R. Gayathri, (D) Harekrushna Behera, et al.
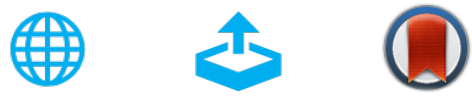

View Online

Export Citation

\section{ARTICLES YOU MAY BE INTERESTED IN}

Water-wave interaction with submerged porous elastic disks

Physics of Fluids 32, 047106 (2020); https://doi.org/10.1063/5.0006119

Bragg scattering of long waves by an array of floating flexible plates in the presence of multiple submerged trenches

Physics of Fluids 32, 096603 (2020); https://doi.org/10.1063/5.0017930

Effect of imposed shear on the dynamics of a contaminated two-layer film flow down a slippery incline

Physics of Fluids 32, 102113 (2020); https://doi.org/10.1063/5.0024201

AlP Author Services

\section{English Language Editing}

High-quality assistance from subject specialists 


\title{
Surface wave scattering by multiple flexible fishing cage system
}

\author{
Cite as: Phys. Fluids 33, 037119 (2021); doi: 10.1063/5.0040662 \\ Submitted: 15 December 2020 • Accepted: 20 February 2021 . \\ Published Online: 19 March 2021

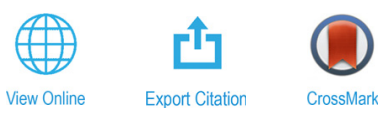

\author{
Siluvai Antony Selvan, ${ }^{1,2, a)}$ (D) R. Gayathri,, ${ }^{2, b)}$ (D) Harekrushna Behera, ${ }^{2, c)}$ (D) and M. H. Meylan ${ }^{3, d)}$ (iD) \\ AFFILIATIONS \\ 'School of Mathematics and Statistics, The University of Melbourne, Parkville VIC 3010, Australia \\ ${ }^{2}$ Department of Mathematics, SRM Institute of Science and Technology, Kattankulathur 603203, Tamil Nadu, India \\ ${ }^{3}$ School of Mathematical and Physical Sciences, University of Newcastle, Callaghan NSW 2308, Australia \\ a) Electronic mail: antony.selvan22@gmail.com \\ b) Electronic mail: gayathri.ramachandran08@gmail.com \\ ${ }^{c)}$ Author to whom correspondence should be addressed: hkb.math@gmail.com \\ ${ }^{d)}$ Electronic mail: mike.meylan@newcastle.edu.au
}

\begin{abstract}
A study of the wave dynamics around a multiple cylindrical fishing cage system is carried out under the assumption of linear water wave theory and small-amplitude wave response. The Fourier-Bessel series expansion of the velocity potential is derived for the regions enclosed under the open-water and cage systems and the immediate vicinity. The scattering between the cages is accounted for by employing Graf's addition theorem. The porous flexible cage system is modeled using Darcy's law and the three-dimensional membrane equation. The edges of the cages are moored along their circumferences to balance its position. The unknown coefficients in the potentials are obtained by employing the matched eigenfunction method. In addition, the far-field scattering coefficients for the entire system are obtained by expanding the Bessel and Hankel functions in the plane wave representation form. Numerical results for the hydrodynamic forces, scattering coefficients, and power dissipation are investigated for various cage and wave parameters. The time simulation for the wave scattering from the cage system is investigated. The study reveals that wave loading on the cage system can be significantly reduced by the appropriate spatial arrangement, membrane tension, and porous-effect parameter. Moreover, the far-field results suggest that the cage system can also be used as a breakwater.
\end{abstract}

Published under license by AIP Publishing. https://doi.org/10.1063/5.0040662

\section{INTRODUCTION}

There is an every growing demand for seafood owing to the population increase (Duarte et al., 2009; Golden et al., 2016). Simultaneously, there is a need to protect the fish stocks in the ocean, especially with the effects of global warming and ocean acidification (McCormick et al., 2013). Both can be made possible using fish farming, which has expanded rapidly in recent decades (Helal et al., 2017; Zhang et al., 2011) in freshwater. To implement this in the open ocean, a series of challenges are posed by the marine environment that must be overcome. Hence, suitable modeling needs to be performed to predict the wave response of such a system. A fish cage can be modeled as a cylindrical perforated membrane-like structure with perforated bottom moored along its circumference (Chan and Lee, 2001). While the wave attenuation by a single flexible porous cage has been well studied (Su et al., 2015; Mandal and Sahoo, 2016), however, there are no studies in the literature regarding the wave response of multiple flexible perforated fishing cage systems, which form the ideal model for a fish farm. This motivates the present study of the hydrodynamic response of a system of multiple fishing cages. In the case of such a cage system, the parameters such as porosity, flexibility, and geometry play a significant role in explaining the wave response of single/multiple cage systems.

In general, the presence of porosity in any off-shore structures can be modeled using Darcy's law (Chwang, 1983; Yu and Chwang, 1994), which damps the high amplitude wave by dissipating its energy. Several studies have investigated the wave dissipation by rigid cylindrical porous structures (Williams et al., 2000; Fredriksson et al., 2003; Sarkar and Bora, 2019a). The permeability, size, and location of the perforation significantly control the hydrodynamic loads acting on the porous cylinder (Williams et al., 2000). The theoretical and 
experimental studies on a porous cylinder performed by Zhao et al. (2011) suggested that there exist additional components corresponding to the porous effect effects along with the wave-radiating damping. In the case of a surface-piercing cylinder, the variation of draft, porosity, and radii influences the wave run-up and hydrodynamic loading on the structure (Sarkar and Bora, 2019b). Sarkar and Bora (2019a) investigated the wave interaction with the surface-piercing bottommounted porous cylinder, where the resonance effect was observed at a particular wavenumber in the presence of porosity. Later, Behera et al. (2020) studied the influence of a truncated concentric porous cylinder on the inner rigid platform, where the optimum number of porous structures for damping the wave amplitude was estimated. Recently, the cnoidal wave diffraction from the dual concentric cylinders in the presence of an arc-shaped outer cylinder was analyzed by Zhai et al. (2020).

In addition to the porosity, the inclusion of flexibility in the offshore structures reduces the damage experienced due to the wave loading. Furthermore, it also proves to be cost-effective and easily portable (Lee and Chen, 1990; Williams et al., 1991; Meylan et al., 1994). The wave passage through the vertical flexible porous membrane was analyzed by Chan and Lee (2001), who incorporated the tensile force in the standard two-dimensional Euler-Bernoulli's equation. They observed that the deformation of structure increases for larger flexibility, and the presence of perforation substantially decreases the deformation. Other than this, the significance of both the porosity and flexibility has also investigated in the case of horizontal floating/submerged two-/three-dimensional structures (Behera and Sahoo, 2015; Meylan et al., 2017; Behera et al., 2018; Selvan et al., 2019; Zheng et al., 2020b). In the case of cylindrical structures, Mandal et al. (2013) studied the effect of a flexible porous cylinder on the inner truncated rigid platform, where the hydrodynamic wave loads were analyzed for various flow and structural parameters. The dynamics of the flexible porous cylinder mounted on the truncated rigid bottom was investigated by Su et al. (2015). The gravity wave interaction with the cylindrical fish cage with a perforated bottom membrane was analyzed by Mandal and Sahoo (2016), and they found that the proper selection of membrane tension along with the cage radius consequently reduces the deflection of the cylindrical sidewall.

In the previous study of wave scattering from a single fishing cage (Su et al., 2015; Mandal and Sahoo, 2016), the bottom of the fish cage was either mounted to a rigid bed or clamped. However, physically, the fishing cage can be moored along its circumferences, which was not addressed in the previous studies of Su et al. (2015) and Mandal and Sahoo (2016). However, there are a few experiments/ numerical simulations based on the multiple net fishing cages (Zhao et al., 2013, 2015 and literature cited therein). In the present study, the surface wave interaction with the multiple fishing cage system is investigated using the eigenfunction expansion and least squares approximation methods. The fishing cages are moored along their cylindrical circumferences using a frictionless linear spring model. In order to consider the scattering between the cages, Grafs addition theorem has been employed. This theorem forms the basis of modeling the wave scattering from multiple floating structures (Kagemoto and Yue, 1986; Park et al., 2012, 2017; Zheng et al., 2020c; Zheng et al., 2020a). Quantities like power dissipation, the reflected and transmitted wave amplitudes at the far-field are analyzed in the present study to investigate the use of multiple fishing cages as a breakwater.
The present manuscript is organized as follows: Sec. II contains the mathematical formulation, where the details of the governing equation and the associated boundary conditions for the physical problem are discussed; Sec. III develops the solution method, where the procedure for solving the physical problem by employing the method of an eigenfunction in conjunction with the technique of least squares approximation is explained in detail. Further, a brief description of the derivation of far-field amplitude function and the power dissipation is given; Sec. IV contains the result and discussion, which is further subdivided into the dual, triple, and multiple fishing cage systems along with a time-domain simulation. In the case of the dual and triple fishing cages, the hydrodynamic wave forces, far-field amplitude functions, and power dissipation are investigated. Moreover, for multiple fishing cage systems, far-field amplitude functions, power dissipation, and flow distributions are analyzed. The technique of time-domain simulation is given in the Subsection IV F. Multimedia figures (Figs. 14-16) show the surface wave scattering by the single and multiple fishing cage system. In Sec. V, the work is summarized, and the significance of the present work is highlighted.

\section{MATHEMATICAL FORMULATION}

The dynamics of surface waves around a system of $\mathrm{N}$ multiple flexible fishing cages [Fig. 1(a)] is studied in the global Cartesian coordinates $(x, y, z)$ with origin overlap with the still water having depth $H$ and $z$-axis pointed upward direction. The position of arbitrary $j$ th and $k$ th fishing cages are marked as $\left(x_{j}, y_{j}\right)$ and $\left(x_{k}, y_{k}\right)$ in the global co-ordinate system, respectively [Fig. 1(b)], which form the origins of the local polar coordinate systems $\left(r_{j}, \theta_{j}, z\right)$ and $\left(r_{k}, \theta_{k}, z\right)$ with $k=1,2, \ldots, \mathrm{N}$ and $j=1,2, \ldots, \mathrm{N}$. The thicknesses of entire cylindrical fishing cages are assumed as $d$, which is assumed very small on comparing with the wavelength of an incident monochromatic wave $(\lambda)$ as a consequence of the small amplitude wave theory. Further, the radii of an array of floating fishing cages are considered as $b_{k}$ with $k=1,2, \ldots, \mathrm{N}$ [Fig. 1(d)]. The incompressible and inviscid fluid is assumed to be having an irrotational and time-harmonic motion of the form $\exp (-\mathrm{i} \omega t)$. The domain enclosed between the still water and a rigid bottom is divided into an exterior and $\mathrm{N}$ interior regions. The general velocity potential is defined as $\Phi(r, \theta, z, t)=\Re\{\phi(r, \theta, z)$ $\left.\mathrm{e}^{-\mathrm{i} \omega t}\right\}$ with $\phi(r, \theta, z)$ be the spatial velocity potential of $k$ th region, where $\omega$ be the angular frequency and $\Re$ denotes the real part. This general potential $\phi(r, \theta, z)$ satisfying all the assumption of fluids obeys the Laplace equation, which can be expressed as

$$
\frac{\partial^{2} \phi}{\partial r^{2}}+\frac{1}{r} \frac{\partial \phi}{\partial r}+\frac{1}{r^{2}} \frac{\partial^{2} \phi}{\partial \theta^{2}}+\frac{\partial^{2} \phi}{\partial z^{2}}=0 .
$$

Furthermore, the spatial velocity potential in the $k$ th region is given as $\phi_{2}^{k}\left(r_{k}, \theta_{k}, z\right)$ and $\phi_{3}^{k}\left(r_{k}, \theta_{k}, z\right)$ with $k=1,2, \ldots, N$. In an outer region, it is denoted by $\phi_{1}\left(r_{k}, \theta_{k}, z\right)$.

The fluid depth enclosing the fishing cage is subdivided into the barrier region $\Lambda_{B}:=\{z \mid-h \leq z \leq 0\}$ and a gap region $\Lambda_{G}:=\{z \mid-H \leq z \leq-h\}$. The normal velocity becomes zero along the rigid bed in both the interior and outer regions, which is given by

$$
\begin{aligned}
& \frac{\partial \phi_{1}}{\partial z}=0, \quad \text { at } \quad z=-H, \\
& \frac{\partial \phi_{3}^{k}}{\partial z}=0, \quad \text { at } \quad z=-H,
\end{aligned}
$$




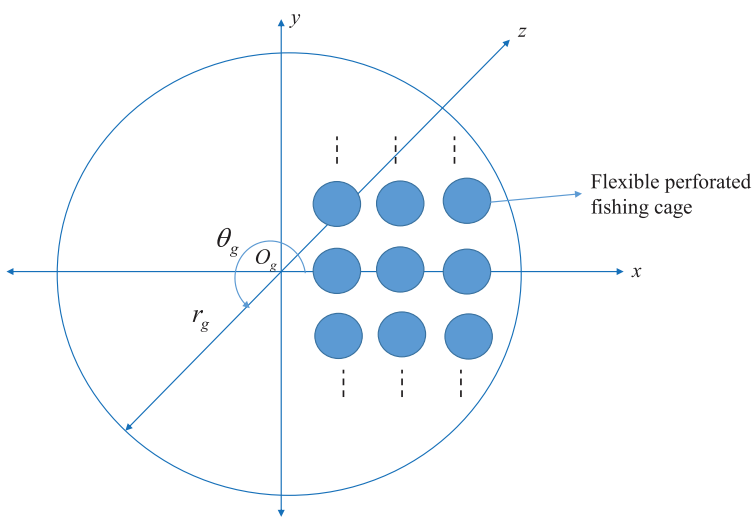

(a)

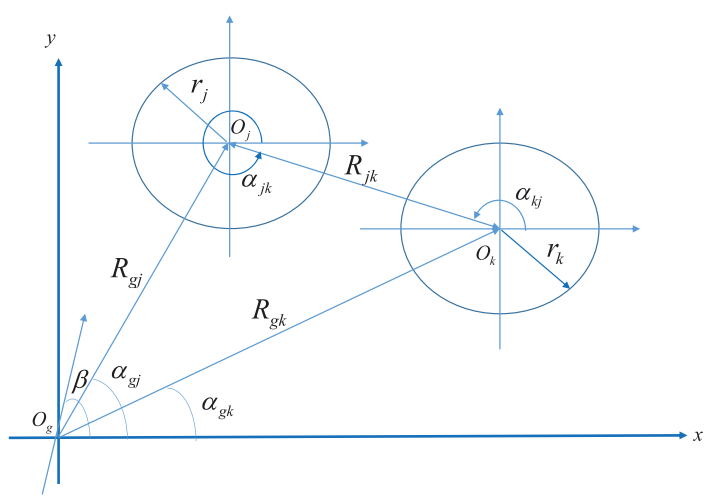

(b)

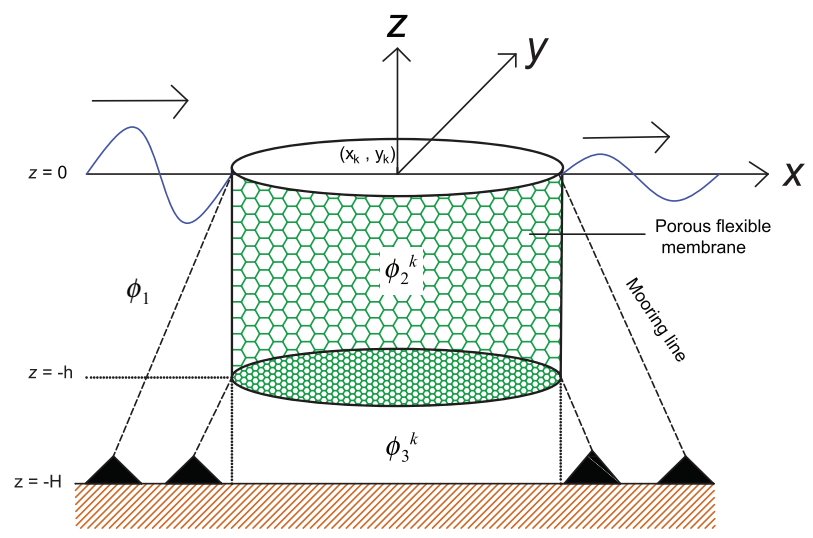

(c)
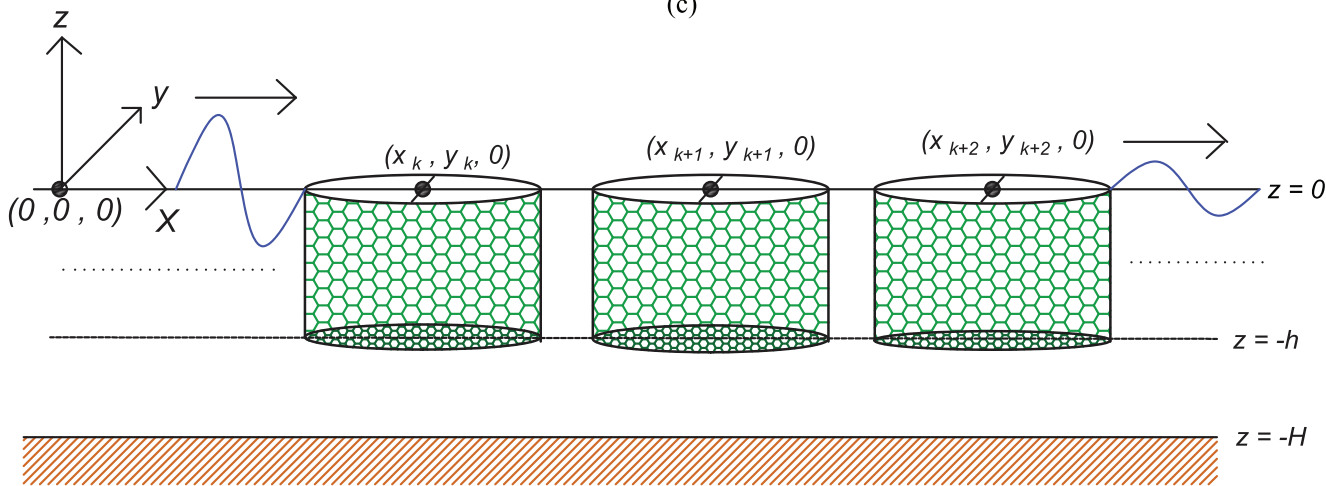

$z=-H$

(d)

FIG. 1. Schematic sketch of the defined physical problem; (a) Top view showing the global cylindrical coordinate $\left(O_{g} r_{g} \theta_{g} z\right)$, (b) top view showing the $k$ th - and jth -cylinders, (c) lateral view of a flexible perforated moored fishing cage, and (d) lateral view showing the array of multiple fishing cages.

where $k=1,2, \ldots \mathrm{N}$. The linear form of the free surface boundary condition at both the open-water and cage covered regions are given as

$$
\begin{aligned}
& \frac{\partial \phi_{1}}{\partial z}-K \phi_{1}=0, \quad \text { at } \quad z=0, \\
& \frac{\partial \phi_{2}^{k}}{\partial z}-K \phi_{2}^{k}=0, \quad \text { at } \quad z=0,
\end{aligned}
$$

where $K=w^{2} / g$ and $g$ is the acceleration due to gravity. Using Darcy's law, the proportionality between the hydrodynamic pressure and the normal velocity along the cage system is obtained, which are given as

$$
\frac{\partial \phi_{1}}{\partial r}=\frac{\partial \phi_{2}^{k}}{\partial r}=\mathrm{i} k_{0} G_{v}\left\{\phi_{2}^{k}-\phi_{1}\right\}+\mathrm{i} \omega \eta_{k}, \quad \text { at } \quad r_{k}=b_{k}, \quad z \in \Lambda_{B},
$$




$$
\frac{\partial \phi_{2}^{k}}{\partial z}=\mathrm{i} k_{0} G_{h}\left\{\phi_{2}^{k}-\phi_{3}^{k}\right\}+\mathrm{i} \omega \zeta_{k}, \quad \text { at } \quad 0<r_{k}<a_{k}, \quad z=-h,
$$

where $G_{v}$ and $G_{h}$ be the complex porous-effect parameters of cylindri$\mathrm{cal}$ and circular membrane, respectively. The deflection of $k$ th cylindrical and submerged membranes are denoted as $\eta_{k}\left(r_{k}, \theta_{k}, z\right)$ and $\zeta_{k}\left(r_{k}, \theta_{k}, z\right)$, respectively. The linear form of a dynamical boundary condition corresponding to the bottom circular membrane reads as (Mandal and Sahoo, 2016)

$$
\begin{aligned}
& \left(Q \frac{\partial^{3} \phi_{3}^{k}}{\partial z^{3}}-m_{m} \frac{\partial \phi_{3}^{k}}{\partial z}-K \phi_{3}^{k}\right) \\
& \quad-\mathrm{i} k_{0} G_{h}\left(Q \frac{\partial^{2} \phi_{3}^{k}}{\partial z^{2}}-m_{m}\right)\left(\phi_{2}^{k}-\phi_{3}^{k}\right)+K \phi_{2}^{k}=0,
\end{aligned}
$$

where $Q=T_{1} / \rho g$ and $m_{m}=\rho_{m} d / \rho g$, with $T_{1}$ and $\rho_{m}$ being the tensile force and density of a bottom perforated circular membrane, respectively. The deflection of $k$ th cylindrical perforated membrane $\eta_{k}\left(r_{k}, \theta_{k}, z\right)$ is governed by (Mandal and Sahoo, 2016)

$$
\begin{gathered}
T_{2} \frac{d^{2} \eta_{k}}{d z^{2}}+m_{s} \omega^{2} \eta_{k}= \\
\text { at } z \in b_{k} \int_{0}^{\pi}\left(\phi_{1}-\phi_{2}^{k}\right) \mathrm{e}^{\mathrm{i}\left(\pi-\theta_{k}\right)} d \theta_{k} \\
\text { and } r=b_{k},
\end{gathered}
$$

where $k=1,2, \ldots \mathrm{N}$. Furthermore, $T_{2}$ and $m_{s}$ are the tensile force and uniform mass per unit length of the cylindrical perforated membrane, respectively. The matching of velocity and pressure along the interface of $k$ th fish cage can be expressed as

$$
\begin{gathered}
\frac{\partial \phi_{1}}{\partial r}=\frac{\partial \phi_{2}^{k}}{\partial r} \quad \text { at } \quad z \in \Lambda_{B} \text { for } \quad r_{k}=b_{k}, \\
\frac{\partial \phi_{1}}{\partial r}=\frac{\partial \phi_{3}^{k}}{\partial r} \quad \text { at } \quad z \in \Lambda_{G} \text { for } \quad r_{k}=b_{k}, \\
\phi_{1}=\phi_{3}^{k}, \quad \text { at } \quad z \in \Lambda_{G} \text { for } \quad r_{k}=b_{k}, \\
\frac{\partial \phi_{2}^{k}}{\partial z}=\frac{\partial \phi_{3}^{k}}{\partial z}, \quad \text { at } \quad r_{k}=b_{k} \quad \text { and } \quad z=-h .
\end{gathered}
$$

Moreover, the condition for mooring along the circumference at both the ends of the cylindrical perforated membrane is given by (Karmakar and Soares, 2012; Selvan and Behera, 2020)

$T_{2} \frac{d \eta_{k}}{d z}-2 \mu \sin ^{2} \gamma_{u} \eta_{k}=0, \quad$ at $\quad z=-h, z=0 \quad$ and $\quad r_{k}=b_{k}$,

$T_{2} \frac{d \eta_{k}}{d z}-2 \mu \sin ^{2} \gamma_{d} \eta_{k}=0, \quad$ at $\quad z=-h, z=0 \quad$ and $\quad r_{k}=b_{k}$,

where $\mu, \gamma_{u}$, and $\gamma_{d}$ are the mooring spring constant, the angle extended by the upper and lower mooring lines with respect to negative $z$-axis, respectively.

\section{METHOD OF SOLUTION}

In the outer region, the velocity potential $\phi_{1}\left(r_{k}, \theta_{k}, z\right)$ satisfying the Laplace equation (1) with the associated bottom boundary condition (2) is derived as

$$
\begin{aligned}
\phi_{1}= & \sum_{m=-\infty}^{\infty}\left(\mathcal{I}_{k} J_{m}\left(k_{0} r_{k}\right) \mathrm{e}^{\mathrm{i} m(\pi / 2-\beta)} g_{0}(z)\right. \\
& \left.+\sum_{k=1}^{\mathrm{N}} \sum_{q=0}^{\infty} A_{m q}^{k} H_{m}^{(1)}\left(k_{q} r_{k}\right) g_{q}(z)\right) \mathrm{e}^{\mathrm{i} m \theta_{k}},
\end{aligned}
$$

where $\mathcal{I}_{k}=(\mathrm{i} g / \omega) \mathrm{e}^{\mathrm{i} k_{0}\left(x_{k} \cos \beta+y_{k} \sin \beta\right)}$ is the phase factor with $\beta$ being the phase angle. Here, $J_{m}$ and $H_{m}^{(1)}$ indicate the Bessel and first kind Hankel functions of order $m$. The unknown coefficients of open-water region for $k$ th fishing cage are denoted by $A_{m q}^{k}$. Further, Eq. (16) satisfies the far-field radiation condition

$$
\lim _{r_{k} \rightarrow \infty} \phi_{1}=\sum_{m=-\infty}^{\infty}\left(\mathcal{I}_{k} J_{m}\left(k_{0} r_{k}\right) \mathrm{e}^{\mathrm{i} m(\pi / 2-\beta)} g_{0}(z)\right),
$$

where the scattered portion of Eq. (16) becomes zero. By employing Grafs addition theorem as in Abramowitz and Stegun (1948) and Zheng et al. (2020a), the Hankel function in the scattered portion of Eq. (16) can be rewritten as

$$
\begin{aligned}
\phi_{1}= & \sum_{m=-\infty}^{\infty}\left[\mathcal{I}_{k} J_{m}\left(k_{0} r_{k}\right) \mathrm{e}^{\mathrm{i} m(\pi / 2-\beta)} \mathrm{e}^{\mathrm{i} m \theta_{k}} g_{0}(z)+\sum_{q=0}^{\infty}\left(A_{m q}^{k} H_{m}^{(1)}\left(k_{q} r_{k}\right) \mathrm{e}^{\mathrm{i} m \theta_{k}}\right.\right. \\
& +\sum_{\substack{j=1 \\
j \neq k}}^{\mathrm{N}} A_{m q}^{j} \sum_{n=-\infty}^{\infty}(-1)^{n} H_{m-n}^{(1)}\left(k_{q} R_{j k}\right) \mathrm{e}^{\mathrm{i}\left(m \alpha_{k j}-n \alpha_{j k}\right)} \\
& \left.\left.\times J_{n}\left(k_{q} r_{k}\right) \mathrm{e}^{\mathrm{i} n \theta_{k}}\right) g_{q}(z)\right]
\end{aligned}
$$

where the eigenfunction $g_{q}(z)$ associated with the outer region is given by

$$
g_{q}(z)=\frac{\cosh k_{q}(z+H)}{\cosh \left(k_{q} H\right)},
$$

in which the eigenvalues $k_{q}$ satisfy the following dispersion relation:

$$
\omega^{2}=g k_{q} \tanh \left(k_{q} H\right),
$$

which can be solved numerically to obtain the one real root $\left(k_{0}\right)$ and infinite number of imaginary roots $\left(k_{1}, k_{2}, k_{3}, \ldots\right)$. Similarly, the velocity potentials corresponding to the interior region of $k$ th fishing cage after solving Eq. (1) along with Eq. (3) are given by

$$
\begin{aligned}
& \phi_{2}^{k}=\sum_{m=-\infty}^{\infty}\left(\sum_{q=0}^{\infty} B_{m q}^{k} I_{m}\left(p_{q} r_{k}\right) M_{q}(z)\right) \mathrm{e}^{\mathrm{i} m \theta_{k}}, \\
& \phi_{3}^{k}=\sum_{m=-\infty}^{\infty}\left(\sum_{q=0}^{\infty} B_{m q}^{k} I_{m}\left(p_{q} r_{k}\right) N_{q}(z)\right) \mathrm{e}^{\mathrm{i} m \theta_{k}},
\end{aligned}
$$

where $I_{m}$ is the modified Bessel function of first kind having the order $m$, and the unknown coefficients of the interior region of $k$ th fishing cage are denoted as $B_{m q}^{k}$. The associated eigenfunctions are given as

$$
\begin{gathered}
M_{q}(z)=\frac{\cosh p_{q}(z+H)-E_{q} \sinh p_{q}(z+H)}{\cosh \left(p_{q} H\right)-E_{q} \sinh \left(p_{q} H\right)}, \\
N_{q}(z)=\frac{\left\{\tanh p_{q}(H-h)-E_{q}\right\}\left\{\cosh p_{q}(z+H)\right\}}{\left\{\tanh p_{q}(H-h)\right\}\left\{\cosh \left(p_{q} H\right)-E_{q} \sinh \left(p_{q} H\right)\right\}},
\end{gathered}
$$


$E_{q}=\frac{V_{q} p_{q} \tanh ^{2} p_{q}(H-h)}{V_{q} p_{q} \tanh p_{q}(H-h)-\left\{1-\tanh ^{2} p_{q}(H-h)\right\}\left\{K-\mathrm{i} k_{0} G_{2} V_{q}\right\}}$,

where $V_{q}=T_{1} p_{q}^{2}-m_{s} w^{2}$. Furthermore, the eigenvalues $p_{q}$ satisfy the following dispersion relation in the interior cage region, which is expressed as:

$$
K-p_{q} \tanh \left(p_{q} H\right)=E_{q}\left(K \tanh \left(p_{q} H\right)-p_{q}\right) .
$$

Solving Eq. (26) numerically yields infinitely many complex roots $\left(p_{0}, p_{1}, p_{2}, \ldots\right)$ for $G \neq 0$. On the other hand, in the case of $G=0$, the one real roots $\left(p_{0}\right)$ and infinitely many imaginary roots $\left(p_{0}, p_{1}, p_{2}, \ldots\right)$ are obtained. The deflection of $k$ th cylindrical membrane obtained by solving Eq. (9) can be represented as

$$
\begin{aligned}
\eta_{k}= & D_{m 1}^{k} \frac{\cos \lambda z}{\cos \lambda_{1} H}+D_{m 2}^{k} \frac{\sin \lambda z}{\sin \lambda_{2} H}+\frac{\mathrm{i} \omega \pi \rho b_{k}}{T_{2}\left(k_{0}^{2}+\lambda^{2}\right)} \\
& \times\left[\sum_{q=0}^{\infty} B_{m q}^{k} I_{m}\left(p_{q} b_{k}\right) M_{q}(z)-\mathcal{I}_{k} J_{m}\left(k_{0} b_{k}\right) e^{\mathrm{i} m(\pi / 2-\beta)} g_{0}(z)\right. \\
& -\sum_{q=0}^{\infty}\left[A_{m q}^{k} H_{m}^{(1)}\left(k_{q} b_{k}\right)+\sum_{j=1 j \neq k^{j=1}}^{\mathrm{N}} \sum_{n=-\infty}^{\infty} A_{n q}^{j} H_{m-n}^{(1)}\left(k_{q} R_{j k}\right)\right. \\
& \left.\left.\times \mathrm{e}^{\mathrm{i}\left(m \alpha_{k j}-n \alpha_{j k}\right)} J_{m}\left(k_{q} b_{k}\right)\right] g_{q}(z)\right],
\end{aligned}
$$

where $\lambda=\sqrt{\frac{m_{s}}{T_{1}}} \omega$. By employing the velocity continuity as in Eqs. (10) and (11), the following the linear system of equation is obtained:

$$
\begin{aligned}
& \mathcal{I}_{k} J^{\prime}{ }_{m}\left(k_{0} b_{k}\right) Y_{l q} \mathrm{e}^{\mathrm{i} m(p i / 2-\beta)} \delta_{l q} \\
& \quad+\sum_{q=0}^{\infty}\left[A_{m q}^{k} H_{m}^{\prime(1)}\left(k_{q} b_{k}\right) Y_{l q} \delta_{l q}+\sum_{\substack{j=1 \\
j \neq k}}^{N} \sum_{n=-\infty}^{\infty} A_{n q}^{j}(-1)^{m}\right. \\
& \quad \times H_{n-m}^{(1)}\left(k_{q} R_{j k}\right) \mathrm{e}^{\mathrm{i}\left(n \alpha_{k j}-m \alpha_{j k}\right)} J_{m}^{\prime}\left(k_{q} b_{k}\right) Y_{l q} \delta_{l q} \\
& -B_{m q}^{k} I_{m}^{\prime}\left(p_{q} b_{k}\right)\left[M_{q l}+N_{q l}\right]=0,
\end{aligned}
$$

where $m=0,1,2, \ldots, l=0,1, \ldots$ and $q=0,1,2, \ldots$ The orthogonal relations $Y_{l}, M_{q}$, and $N_{q l}$ with respect to the open-water region are given by

$$
\begin{gathered}
Y_{l q}=\int_{-H}^{0} g_{l}(z) g_{q}(z) d z, \quad M_{q l}=\int_{-h}^{0} M_{q}(z) g_{l}(z) d z, \\
\text { and } N_{q l}=\int_{-H}^{-h} N_{q}(z) g_{l}(z) d z,
\end{gathered}
$$

with $q=0,1, \ldots$ and $l=0,1, \ldots$ Using Eqs. (6) and (12), the coupled relation is obtained for $k=1,2, \ldots, \mathrm{N}$, which is given by

$$
\begin{aligned}
X^{k}(z)= & \sum_{q=0}^{\mathbf{n}_{0}}\left[A_{m q}^{k} R_{m q}^{k}(z)+\sum_{\substack{j=1 \\
j \neq k}}^{N} \sum_{n=0}^{M} A_{n q}^{j} R_{n q}^{j}(z)\right] \\
& +\sum_{q=0}^{\mathbf{n}_{0}} B_{m q}^{k} S_{m q}^{k}(z)+\sum_{i=1}^{2} D_{m i}^{k} W_{m i}^{k}(z)+L_{m 0}^{k}(z),
\end{aligned}
$$

where

$$
\begin{aligned}
& R_{m q}^{k}(z)=\left\{\begin{array}{l}
H_{m}^{(1)}\left(k_{q} b_{k}\right) g_{q}(z) \quad z \in \Lambda_{G}, \\
\left(\frac{b_{k} \omega^{2} \rho \pi}{T_{2}\left(k_{0}^{2}+\lambda^{2}\right)}-\mathrm{i} k_{0} G_{v}\right) H_{m}^{(1)}\left(k_{q} b_{k}\right) g_{q}(z) \quad z \in \Lambda_{B},
\end{array}\right. \\
& R_{n q}^{j}(z)=\left\{\begin{array}{l}
H_{n-m}^{(1)}\left(k_{q} R_{j k}\right) \mathrm{e}^{\mathrm{i}\left(n \alpha_{k j}-m \alpha_{j k}\right)} J_{m}\left(k_{q} b_{k}\right) g_{q}(z) \quad z \in \Lambda_{G}, \\
\left(\frac{b_{k} \omega^{2} \rho \pi}{T_{2}\left(k_{q}^{2}+\lambda^{2}\right)}-\mathrm{i} k_{0} G_{v}\right) H_{n-m}^{(1)}\left(k_{q} R_{j k}\right) \\
\quad \times \mathrm{e}^{\mathrm{i}\left(n \alpha_{k j}-m \alpha_{j k}\right)} J_{m}\left(k_{q} b_{k}\right) g_{q}(z) \quad z \in \Lambda_{B},
\end{array}\right. \\
& S_{m q}^{k}(z) \\
& =\left\{\begin{array}{l}
-I_{m}\left(p_{q} b_{k}\right) N_{q}(z) z \in \Lambda_{G}, \\
\left(\left(-\frac{b_{k} \omega^{2} \rho \pi}{T_{2}\left(p_{q}^{2}+\lambda^{2}\right)}-\mathrm{i} k_{0} G_{v}\right) I_{m}\left(p_{q} b_{k}\right)-I_{m}^{\prime}\left(p_{q} b_{k}\right)\right) M_{q}(z) z \in \Lambda_{B},
\end{array}\right. \\
& L_{m 0}^{k}(z)=\left\{\begin{array}{l}
\mathcal{I}_{k} J_{m}\left(k_{0} b_{k}\right) \mathrm{e}^{\mathrm{i} m(\pi / 2-\beta)} g_{q}(z) z \in \Lambda_{G}, \\
\left(\frac{b_{k} \omega^{2} \rho \pi}{T_{2}\left(k_{0}^{2}+\lambda^{2}\right)}-\mathrm{i} k_{0} G_{v}\right) \mathcal{I}_{k} J_{m}\left(k_{0} b_{k}\right) \mathrm{e}^{\mathrm{i} m(\pi / 2-\beta)} g_{q}(z) z \in \Lambda_{B},
\end{array}\right. \\
& W_{m 1}^{k}(z)= \begin{cases}0 & z \in \Lambda_{G}, \\
\frac{\mathrm{i} \omega \cosh (\lambda z)}{\cosh (\lambda H)} & z \in \Lambda_{B},\end{cases} \\
& W_{m 2}^{k}(z)=\left\{\begin{array}{l}
0 \quad z \in \Lambda_{G}, \\
\frac{\mathrm{i} \omega \sinh (\lambda z)}{\cosh (\lambda H)} \quad z \in \Lambda_{B} .
\end{array}\right.
\end{aligned}
$$

The above relation Eq. (30) satisfies the least squares approximation method as given by

$$
\begin{gathered}
\int_{-H}^{0}\left|X^{k}\right|^{2} d z=\text { minimum } \\
\int_{-H}^{0} X^{k} \frac{\partial X^{k *}}{\partial A_{m q}^{k *}} d z=0
\end{gathered}
$$

where $*$ denotes the complex conjugate. The series expansion for eigenvalues is truncated for $q=\mathbf{n}_{0}$ roots for both the open water and submerged membrane covered regions for $k$ th fishing cage. Similarly, the Bessel series are truncated for $m=M$ for $k$ th fishing cage. This leads to $2 N M\left(\mathbf{n}_{0}+1\right)$ system of equations [Eqs. (28) and (30) along with the edge conditions (14) and (15)], which can be solved for all values of $m, q$, and $k$ simultaneously.

\section{A. Far-field amplitude functions and power dissipation}

As $r_{k} \rightarrow \infty$, there exists only the progressive wave mode in the scattered part of the total potential Eq. (16) corresponding to the exterior region, which can be expressed in the following asymptotic form:

$$
\phi_{1}^{s}=\sum_{k=1}^{N} \sum_{m=-\infty}^{\infty} A_{m 0}^{k} \sqrt{\frac{2}{\pi k_{0} r_{k}}} e^{-\mathrm{i} m \pi / 4} e^{\mathrm{i} k_{0} r_{k}} e^{\mathrm{i} m\left(\theta_{k}-\pi / 2\right)} g_{0}(z) .
$$

Furthermore, the above expression, Eq. (33), is transformed from the local cylindrical co-ordinate $\left(r_{k}, \theta_{k}, z\right)$ to the global cylindrical coordinate $\left(r_{g}, \theta_{g}, z\right)$, which can be rewritten as 


$$
\phi_{1}^{s}=\sum_{k=1}^{N} \sum_{m=-\infty}^{\infty} A_{m 0}^{k} \sqrt{\frac{2}{\pi k_{0} r_{k}}} e^{-\mathrm{i} m \pi / 4} e^{\mathrm{i} k_{0} R_{g k} \cos \left(\alpha_{g k}-\theta_{g}\right)} e^{\mathrm{i} m\left(\theta_{g}-\pi / 2\right)} g_{0}(z) .
$$

Similarly, the incident portion of the total potential Eq. (16) is expressed in the asymptotic form and extended to the global cylindrical coordinate, which is given as

$$
\phi_{1}^{i n}=\sum_{m=-\infty}^{\infty} \mathcal{I}_{k} e^{-\mathrm{i} k_{0} R_{g k} \cos \left(\alpha_{g k}-\theta_{g}\right)} e^{\mathrm{i} m(\pi / 2-\beta)} g_{0}(z)
$$

From Eqs. (34) and (35), the scattering and incident amplitude functions are denoted as

$$
\begin{gathered}
\mathbf{D}\left(\theta_{g}\right)=\frac{\mathrm{i} \omega}{g \pi} \sum_{k=1}^{N} \sum_{m=-\infty}^{\infty} A_{m 0}^{k} e^{-\mathrm{i} k_{0} R_{g k} \cos \left(\alpha_{g k}-\theta_{g}\right)} e^{\mathrm{i} m\left(\theta_{g}-\pi / 2\right)} \\
\text { and } \mathbf{A}\left(\theta_{g}\right)=\frac{A}{2 \pi} \sum_{m=-\infty}^{\infty} e^{\mathrm{i} m\left(\theta_{g}-\beta\right)} .
\end{gathered}
$$

Further, the power dissipation is obtained directly from the hydrodynamic pressure acting on the vertical and submerged porous membrane, which is given as

$$
\begin{aligned}
\mathcal{P}_{D}= & \frac{k_{0} \rho \omega}{2}\left[\left(G_{v}+G_{v}^{*}\right) \sum_{k=1}^{N} \int_{\Omega_{h}}\left|\phi_{2}^{k}-\phi_{3}^{k}\right|^{2} d S\right. \\
& \left.+\left(G_{h}+G_{h}^{*}\right) \sum_{k=1}^{N} \int_{\Omega_{v}}\left|\phi_{2}^{k}-\phi_{1}\right|^{2}\right] d S,
\end{aligned}
$$

where $\Omega_{v}=\left\{(\theta, z): 0 \leq \theta_{k} \leq 2 \pi\right.$ and $\left.-h \leq z \leq 0\right\} \quad$ and $\quad \Omega_{h}$ $=\left\{(r, \theta): 0 \leq r \leq b_{k}\right.$ and $\left.0 \leq \theta_{k} \leq 2 \pi\right\}$ are the domains of integration, and $*$ denotes the complex conjugate. The incident power per unit width of wave front is given as

$$
\mathcal{P}_{I}=\frac{\rho \omega g A^{2}}{4 k_{0}}\left(1+\frac{2 k_{0} H}{\sinh \left(2 k_{0} H\right)}\right),
$$

which can be used for scaling the power dissipation $\mathcal{P}_{D}$. Hence, the non-dimensional power dissipation is denoted as $E=k_{0} \mathcal{P}_{D} / \mathcal{P}_{I}$.

\section{RESULT AND DISCUSSION}

In this section, the effects of a single and double fishing cage system on the surface wave scattering are analyzed for various physical parameters. Moreover, the wave scattering from a multiple fishing cage system is investigated for different spatial configurations. For numerical computation, the following physical parameters such as water depth $H=30 \mathrm{~m}$, density of water $\rho=1025 \mathrm{kgm}^{-3}$, wave amplitude $A=1 \mathrm{~m}$, height of fish cage $h / H=0.5$, density of submerged membrane $\rho_{m}=100 \mathrm{kgm}^{-3}$, non-dimensional tensile force $T_{1} / \rho g H^{2}$ $=T_{2} / \rho g H^{2}=T$ with $T=0.4$, non-dimensional mass of cylindrical cage $\alpha=0.01$, porous-effect parameter of cage system $G_{h}=G_{v}$ $=G=3+3 \mathrm{i}$, mooring spring constant for mooring lines connected to both the ends of cage $\mu=10^{12} \mathrm{~N} / \mathrm{m}$, mooring angles $\gamma_{u}=60^{\circ}$ and $\gamma_{d}=30^{\circ}$, and phase angle $\beta=0^{\circ}$ are fixed, unless it is highlighted in the appropriate figure's caption. The hydrodynamic wave load acting on a fishing cage in vertical direction $\mathcal{S}_{v}$ can be expressed as

$$
\begin{aligned}
& \mathcal{S}_{v}= \mathrm{i} \rho \omega \int_{0}^{2 \pi} \int_{0}^{b_{k}}\left[\phi_{3}^{k}\left(r_{k}, \theta_{k},-h\right)-\phi_{2}^{k}\left(r_{k}, \theta_{k},-h\right)\right] \\
& \times r_{k} d r_{k} \cos \left(\pi-\theta_{k}\right) d \theta_{k}, \\
& \mathcal{S}_{h}=\mathrm{i} \rho \omega \int_{0}^{2 \pi} \int_{0}^{h}\left[\phi_{2}^{k}\left(b_{k}, \theta_{k}, z\right)-\phi_{1}\left(b_{k}, \theta_{k}, z\right)\right] d z \cos \left(\pi-\theta_{k}\right) d \theta_{k} .
\end{aligned}
$$

Furthermore, the non-dimensional form of vertical wave force can be represented as

$$
C_{v}=\left|\frac{\mathcal{S}_{v}}{\rho g b_{k} h H}\right| \quad \text { and } \quad C_{h}=\left|\frac{\mathcal{S}_{h}}{\rho g b_{k} h H}\right| .
$$

\section{A. Numerical validation}

The non-dimensional horizontal wave force $\left(C_{h}\right)$ as a function of non-dimensional radius $\left(b_{1} / H\right)$ is plotted for various edge conditions in the case of a single fishing cage [Fig. 2(a)]. The physical parameters are taken the same as in Mandal and Sahoo (2016) for validating the present theory. In the case of fishing cage clamped on both ends, the reproduced result is in well-agreement with the result of Mandal and Sahoo (2016). It is noticed that the horizontal wave force increases with increasing radius and attains the optimum value, then decreases gradually for larger $b_{1} / H$ for a clamped edge. However, in the presence of mooring spring (either at both ends or one of the ends), the horizontal wave force attains the two optima for varying radius of the cage. It may be due to the stiffness of the linear mooring spring and cage tension. On the other hand, the role of porosity in the horizontal wave force exerted on the cage system for varying $b_{1} / H$ is plotted in Fig. 2(b). The patterns are similar to the observation made in Fig. 2(a) for the moored fishing cage. The variation of wave force behaves differently for smaller radii compared to the larger radii (i.e., the wave force increasing for an increase in $G$ for smaller radii, whereas it shows an opposite trend for larger radii). This occurs due to the enlarged surface area of the cage, where more waves are dissipated. In the case of small radii, the surface area is insufficient for dissipating more waves for larger $G$, and it increases the hydrodynamic wave load on the cage by transmitting more waves inside the cage.

\section{B. Single fishing cage}

Figure 3 portrays the amplitude of wavefield in both the presence and absence of a fishing cage. In the first window [Fig. 3(a)], the incident wave propagating with the phase angle $\beta=30^{\circ}$ and unit peak-to-peak amplitude is illustrated. When it interacts with the single fishing cage as seen in Figs. 3(b)-3(d), the waves get scattered. A portion of waves is dissipated due to the presence of porosity in the cage. Further, the amplitude of scattered wavefield is less than the absolute value of incident amplitude (i.e., $|A|<2$ ). On increasing the nondimensional wavenumber $k_{0} h$, the wave energy decreases in the deep water. Thus, in the presence of a fishing cage, more energy dissipation occurs at larger values of $k_{0} h$. It is clear from Fig. 3(a) that the waves with the smaller wavenumbers are damped more efficiently as compared to the waves having larger wavenumbers [Figs. 3(b)-3(d)].

Figures 4(a)-4(c) illustrate the energy dissipation as a function of real porous-effect parameters [where the inertial effects, i.e., $\operatorname{Im}(G)=0$ are neglected] for different values of $T$ at various wavenumbers. In 


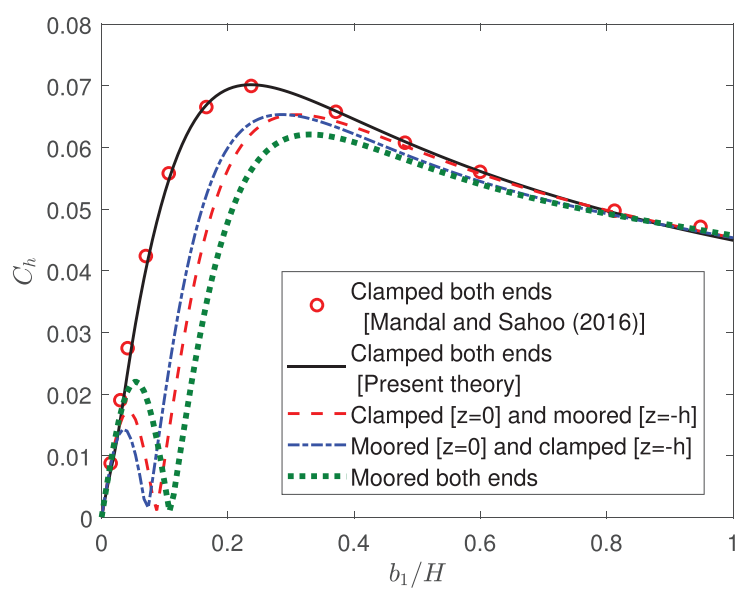

(a)

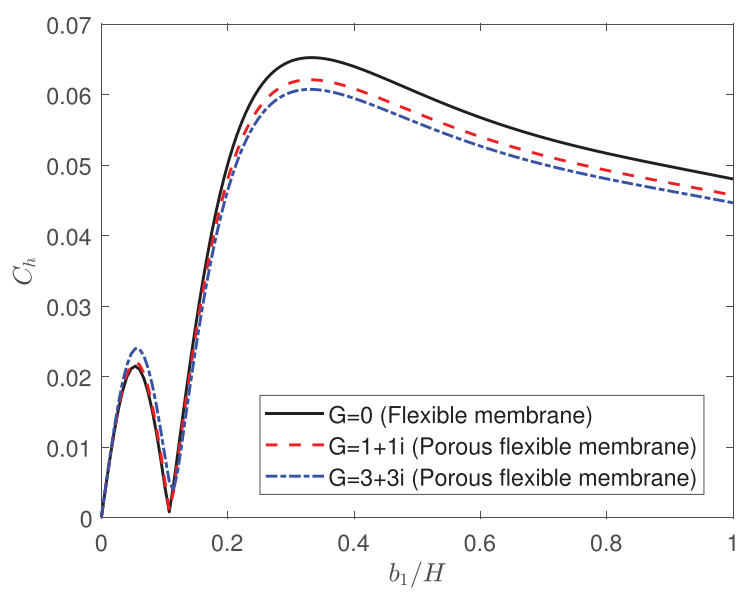

(b)

FIG. 2. Non-dimensional horizontal wave force against non-dimensional radius $\left(b_{1} / H\right)$ for different (a) edge conditions with $G=1+1 i$ and (b) porous effect parameters $G$ with mooring at both ends. The constant parameters are $H=10 \mathrm{~m}, h / H=0.5, k_{0} H=3, \mu=10^{7} \mathrm{~N} / \mathrm{m}, \gamma_{u}=60^{\circ}, \gamma_{d}=35^{\circ}$, and $\alpha=0.1$.

general, the energy dissipation increases initially and attains maximum, then decreases for larger values of $G$. There is a reduction of wave energy dissipation for larger values of $G$ due to the cage's transparency to the incoming wave, where dissipation does not occur. With an increase in the values of $T$, the dissipation follows an increasing pattern for smaller $G$, and it follows the reverse pattern for moderate and larger $G$. When the porous-effect parameter is less, the increasing tensile force develops more restoring force against the wave interacting with the cage. Thus, it dissipates more energy. On the other hand, for moderate and larger $G$, the structure gradually becomes transparent, and the waves are more likely reflected than dissipated. This is the reason for the decreasing energy dissipation for moderate and larger values of $G$. For the waves with larger wavenumber [Fig. 4(c)], the energy dissipation increases for smaller and moderate values of $G$, whereas it falls significantly for larger values of $G$.

The modulus of scattering coefficient along the different directions from the single fishing cage is plotted in Fig. 5 for different porous-effect parameter $G$ at various spring constant $\mu$. The angle at which the surface waves scattered are denoted by $\theta_{g}$. It is observed that the scattering coefficient peaks along the incident wave direction (i.e., $\beta=30^{\circ}$ ). There exist two sidebands on either side of the central band, which indicate that the wave scattering decreases periodically on either side of the incident wave direction. On increasing the mooring spring constant, the membrane stretches more, and the wave scattering decreases as a consequence of less oscillation by a moored cylindrical membrane. On increasing the porous-effect parameter, the wave scattering decreases owing to an increasing wave energy dissipation. However, the deviations are quite significant for smaller and moderate values of $\mu$. In the case of larger $\mu$ [Fig. 5(c)], the effect of $G$ becomes negligible, and the sideband dampens, which is due to less scattering and dissipation by a membrane having very high stiffness. On the other hand, the modulus of the scattering coefficient is plotted in Fig. 6 for different phase angles $\beta$ at various mooring angles $\gamma_{d}$. For a different phase angle, it is noticed that the waves are scattered along a different incident wave direction. The maximum scattering occurs along a specific $\theta_{g}$, which corresponds to the incident phase angle $\beta$. Moreover, the scattering coefficient attains minimum value for larger values of $\gamma_{d}$, implying the occurrence of minimum scattering at larger $\gamma_{d}$.

In Figs. $7(\mathrm{a})-7$ (c), the vertical wave force acting on the fishing cage is plotted as a function of non-dimensional wavenumber $k_{0} h$ for different values of $G$ at $\mu=10^{5} \mathrm{~N} / \mathrm{m}, \mu=10^{6} \mathrm{~N} / \mathrm{m}$, and $\mu=10^{7} \mathrm{~N} / \mathrm{m}$, respectively. The vertical wave force increases and attains maximum, then decreases for increasing values of $k_{0} h$. The maximum wave force happens at a certain wavenumber as a result of increased wave reflection from the cage, in which the dissipation decreases. This specific wavenumber shifts based on the cage parameter. With an increase in the mooring spring constant, the magnitude of wave force increases initially; then, there is a moderate decrease for increasing $\mu$. It is interpreted that there exists an optimum value of mooring spring constant (i.e., $\mu=10^{6} \mathrm{~N} / \mathrm{m}$ ), where the wave force attains maximum value resulting in more reflection. Further, there is a sharp rise in the wave force for increasing $\mu$, which is due to the increased membrane stiffness.

\section{Dual fishing cage}

In Fig. 8, the effect of spacing on the amplitude of surface elevation is plotted. It is noticed that the wave interacts the dual system with phase angle $\beta=30^{\circ}$ in both the figures. On interacting the first cage, the wave amplitude inside the cage increases initially, then there is an energy loss that happens inside the cage due to the destructive interference between the incoming waves and inner scattered waves. The same phenomenon of destructive interference occurs inside the second cage. However, the constructive interference occurs between the cages on keeping both cages close to each other (i.e., $R_{12}=H$ ). This results in the high amplitude waves in the region confined between the two cages as observed in Fig. 8(a). For larger spacing between the cages (i.e., $R_{12}=2 H$ ), there is a loss in wave energy resulting in the damping of wave amplitudes between the cages as observed in Fig. 8(b). Moreover, on comparing both the figures, the wave propagation in the lee-ward side of the structure decreases when 


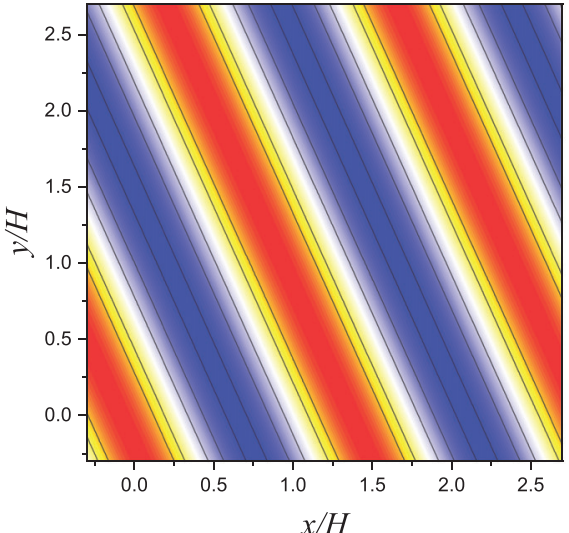

(a) Absence of cage $\left(k_{0} h=1.25\right)$

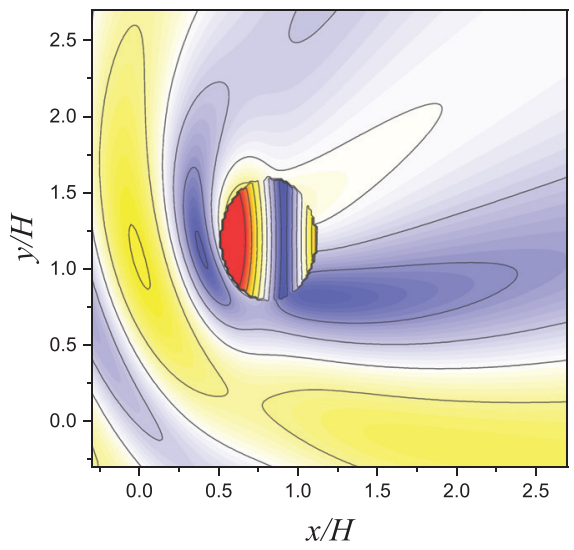

(c) Presence of cage $\left(k_{0} h=1.25\right)$
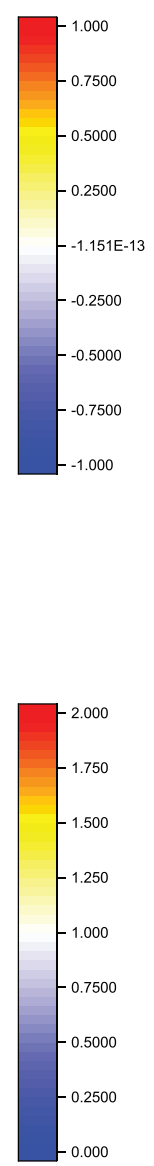

(d) Presence of cage $\left(k_{0} h=1.5\right)$

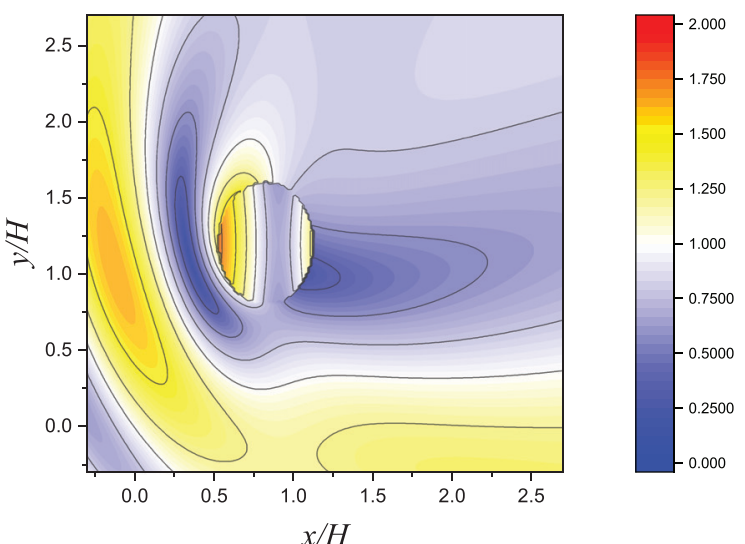

(b) Presence of cage $\left(k_{0} h=1\right)$

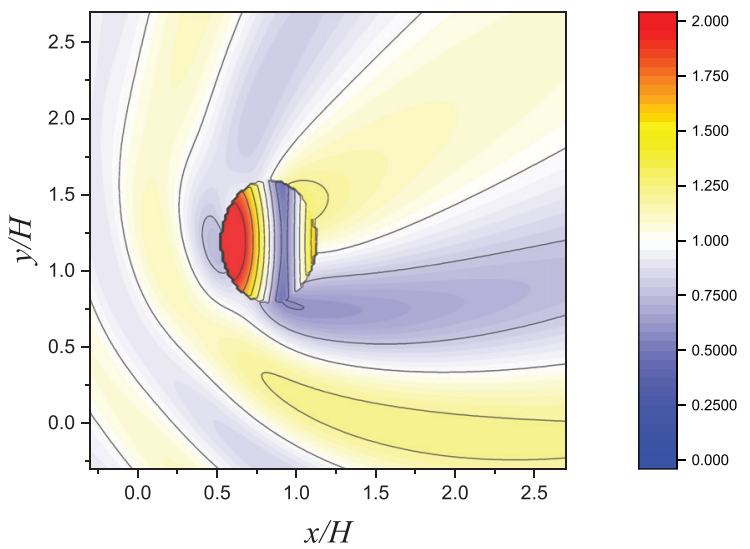

FIG. 3. Surface amplitude distributions $|\eta|$ for the (a) absence and (b)-(d) presence of fishing cage at different non-dimensional wavenumber (a) $k_{0} h=1.25$, (b) $k_{0} h=1,(\mathrm{c})$ $k_{0} h=1.25$, and (d) $k_{0} h=1.5$. The other parameters are $G=3+3 i, T=0.4, h / H=0.4, \beta=30^{\circ}$, and $\mu=10^{6} \mathrm{~N} / \mathrm{m}$.

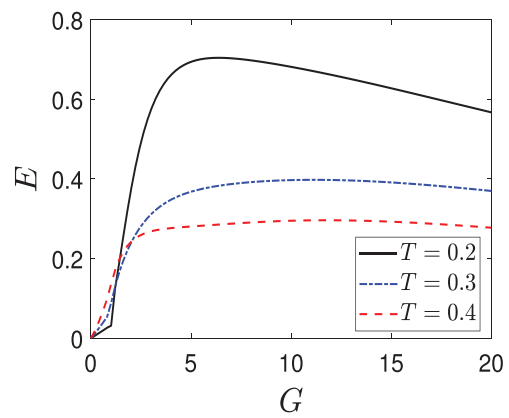

(a) $k_{0} h=1$

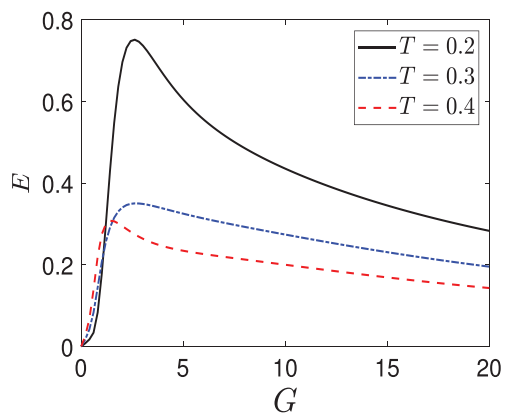

(b) $k_{0} h=1.25$

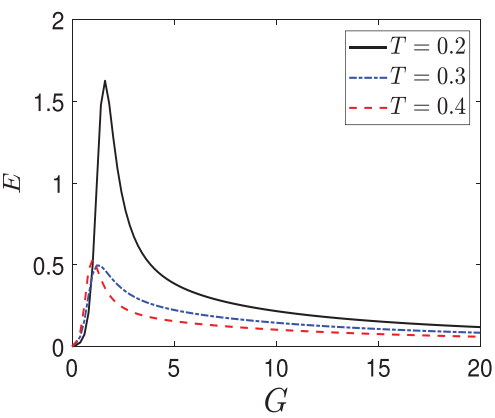

(c) $k_{0} h=1.5$

FIG. 4. Power dissipation $E$ against the real part of $G$ for different values of $T$ at various nondimensional wavenumbers (a) $k_{0} h=1$, (b) $k_{0} h=1.25$, and (c) $k_{0} h=1.5$. The other parameters are $\mathrm{G}=3+3 \mathrm{i}, h / H=0.4, \beta=30^{\circ}$, and $\mu=10^{6} \mathrm{~N} / \mathrm{m}$. 


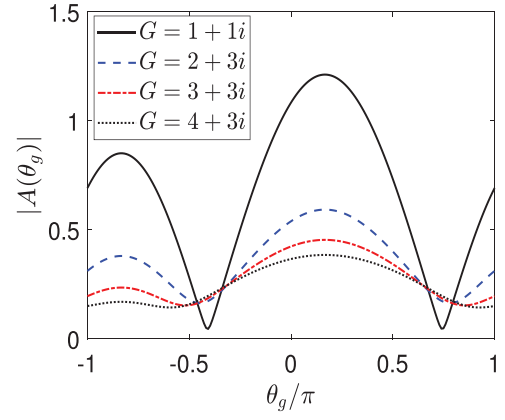

(a) $\mu=10^{5} \mathrm{~N} / \mathrm{m}$

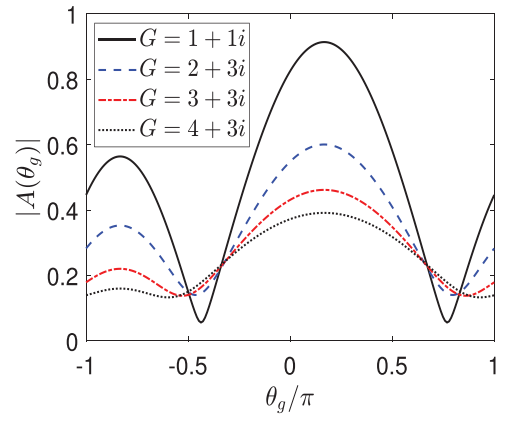

(b) $\mu=10^{6} \mathrm{~N} / \mathrm{m}$

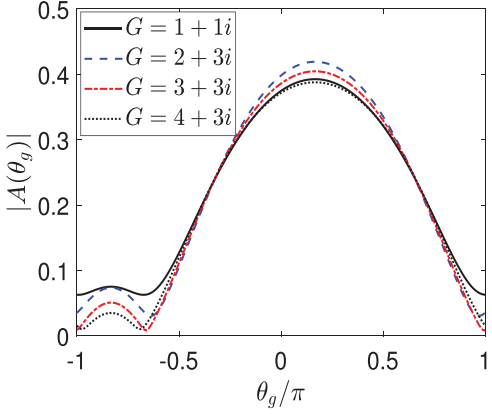

(c) $\mu=10^{7} \mathrm{~N} / \mathrm{m}$

FIG. 5. Modulus of scattering coefficient $\left|A\left(\theta_{g}\right)\right|$ for different values of $G$ at various mooring spring constant (a) $\mu=10^{5} \mathrm{~N} / \mathrm{m}$, (b) $\mu=10^{6} \mathrm{~N} / \mathrm{m}$, and (c) $\mu=10^{7} \mathrm{~N} / \mathrm{m}$. The other parameters are $T=0.4, h / H=0.4, \beta=30^{\circ}$, and $k_{0} h=1.25$.

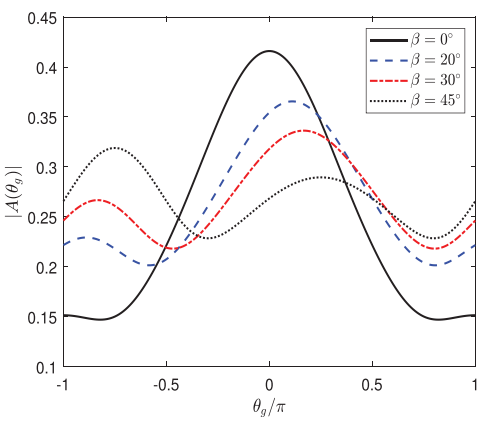

(a) $\gamma_{d}=20^{\circ}$

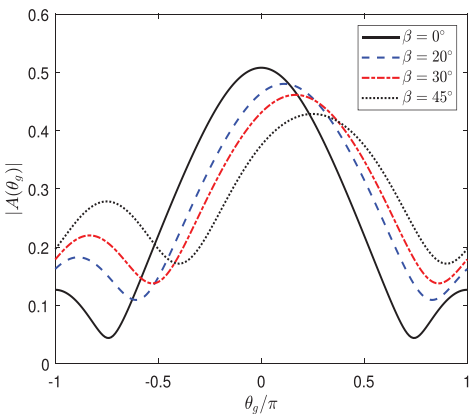

(b) $\gamma_{d}=30^{\circ}$

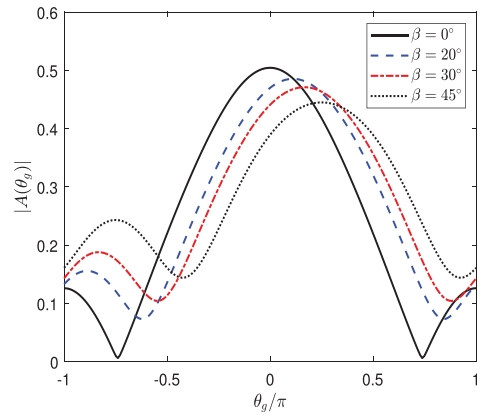

(c) $\gamma_{d}=45^{\circ}$

FIG. 6. Modulus of scattering coefficient $\left|A\left(\theta_{g}\right)\right|$ for different values of $\beta$ at various mooring angle (a) $\gamma_{d}=20^{\circ}$, (b) $\gamma_{d}=30^{\circ}$, and (c) $\gamma_{d}=45^{\circ}$. The other parameters are $T=0.4, h / H=0.4, \mu=10^{6} \mathrm{~N} / \mathrm{m}$, and $k_{0} h=1.25$.

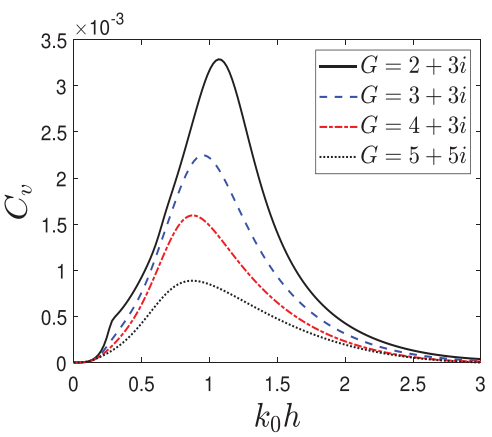

(a) $\mu=10^{5} \mathrm{~N} / \mathrm{m}$

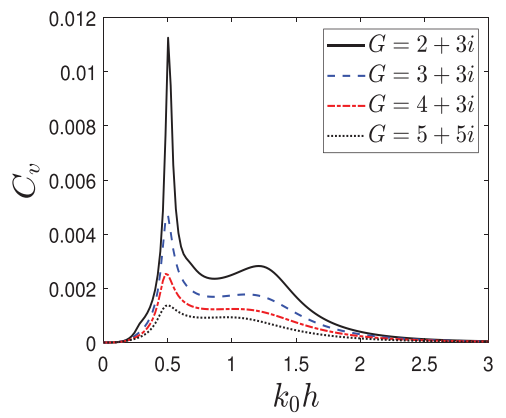

(b) $\mu=10^{6} \mathrm{~N} / \mathrm{m}$

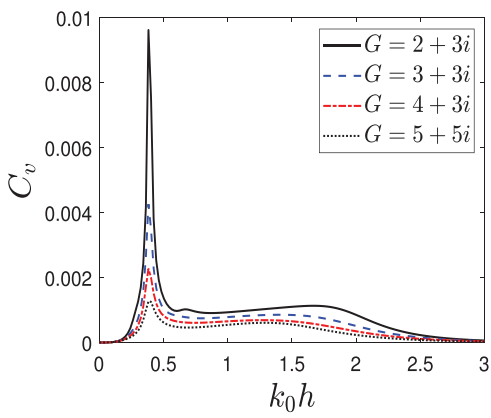

(c) $\mu=10^{7} \mathrm{~N} / \mathrm{m}$

FIG. 7. Vertical wave force acting on the fishing cage against the wavenumber for different values of $\mathrm{G}$ at various mooring spring constant (a) $\mu=10^{5} \mathrm{~N} / \mathrm{m}$, (b) $\mu=10^{6} \mathrm{~N} / \mathrm{m}$, and (c) $\mu=10^{7} \mathrm{~N} / \mathrm{m}$. The other parameters are $T=0.4, h / H=0.4, \beta=30^{\circ}$, and $k_{0} h=1.25$.

the spacing between the cages increases. This, in turn, reduces the damages to the fishing cage system.

The modulus of scattering coefficient along the different directions from the dual fishing cage system is plotted in Fig. 9 for different porous-effect parameter $G$ at various tensile force $T$. It is noticed that the central band peaks at the incident wave direction for $\theta_{g}=30^{\circ}$ in all figures. For smaller tensile force [Fig. 9(a)], the central band reduces and scatters less wave as compared to the sidebands, which scatters 


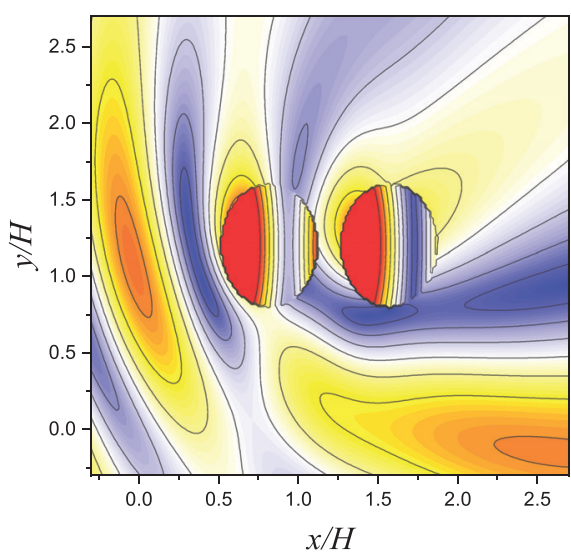

(a) $R_{12}=H$
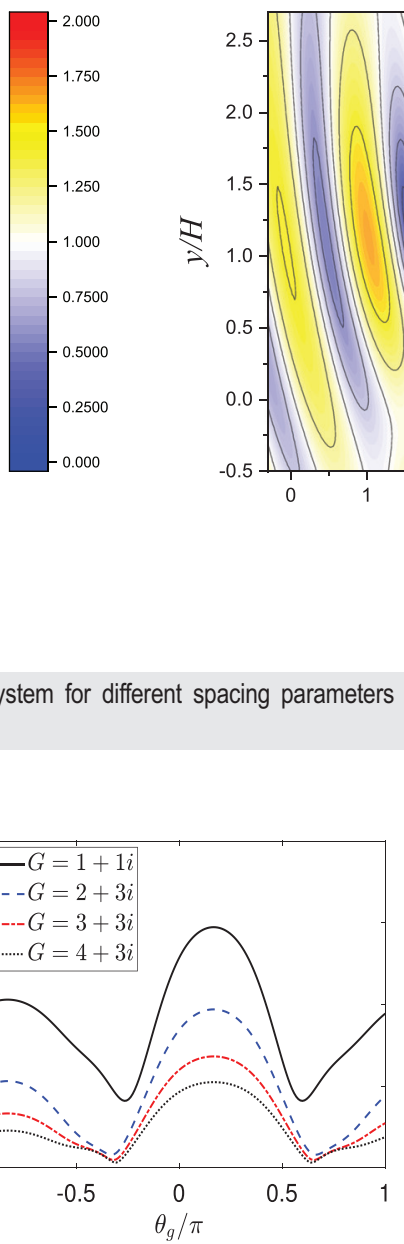

(b) $T=0.2$

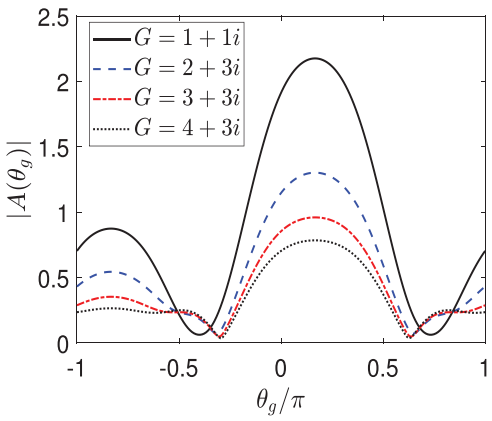

(c) $T=0.4$

FIG. 9. Modulus of scattering coefficient $\left|A\left(\theta_{g}\right)\right|$ for different values of $G$ at various tensile force (a) $T=0.1$, (b) $T=0.2$, and (c) $T=0.4$. The other parameters are $h / H=0.4, \beta=30^{\circ}, k_{0} h=1.25, R_{12}=H$, and $\mu=10^{6} \mathrm{~N} / \mathrm{m}$.

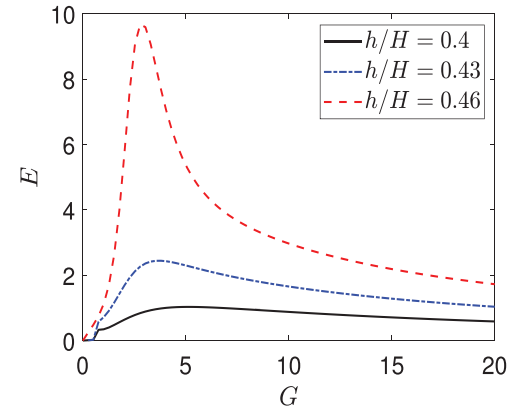

(a) $T=0.1$

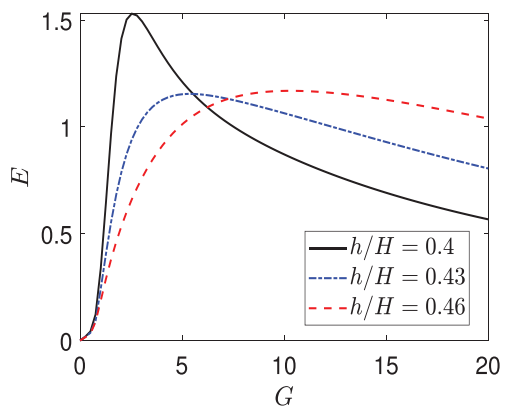

(b) $T=0.2$

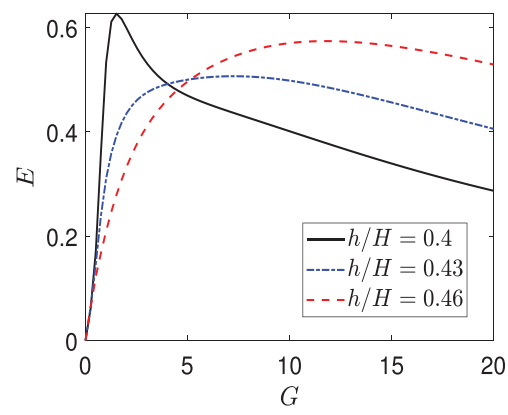

(c) $T=0.4$

FIG. 10. Power dissipation $E$ against the real part of $G$ for different values of $h / H$ at various tensile force (a) $T=0.1$, (b) $T=0.2$, and (c) $T=0.4$. The other parameters are $G=3+3 \mathrm{i}, \beta=30^{\circ}, k_{0} h=1.25, R_{12}=H$, and $\mu=10^{6} \mathrm{~N} / \mathrm{m}$. 
more waves. As the value of $T$ increases, the central band increases, and the sideband decreases owing to the increasing restoring force developed inside the membrane. Moreover, there exists an optimum value of $T$ (i.e., $T=0.2$ ) at which the maximum waves are scattered, after which it decreases slowly for increasing $T$. On increasing the value of $G$, the wave scattering from the cage system increases as a consequence of impedance offered by the cages for membrane having less tensile force. For $T \geq 0.2$, the waves scatter from the dual cage system decrease for an increasing porous-effect parameter, where the impedance decreases and wave energy dissipation increases.

The energy dissipation by the dual fishing cages against the real values of $G$ is plotted for various $h / H$ at varying $T$ in Fig. 10, respectively. Initially, the power dissipation increases with respect to $G$, then it attains maximum and befalls gradually for larger values of $G$. It is noticed that for $T=0.1$, the energy dissipation increases for an increasing $h / H$ as a result of increasing energy dissipation due to the increased surface area of the cage. In the case of $T \geq 0.2$, the energy dissipation behaves differently for $G<6$ and $G>6$. In the case of $G<6$, the energy dissipation decreases for increasing submergence depth $h / H$, where more scattering occurs as compared to the dissipation. On the other hand, for $G>6$, the energy dissipation increases for increasing $h / H$ resulting in less wave scattering as compared to the energy dissipation. Further, the membrane with less tension dissipates

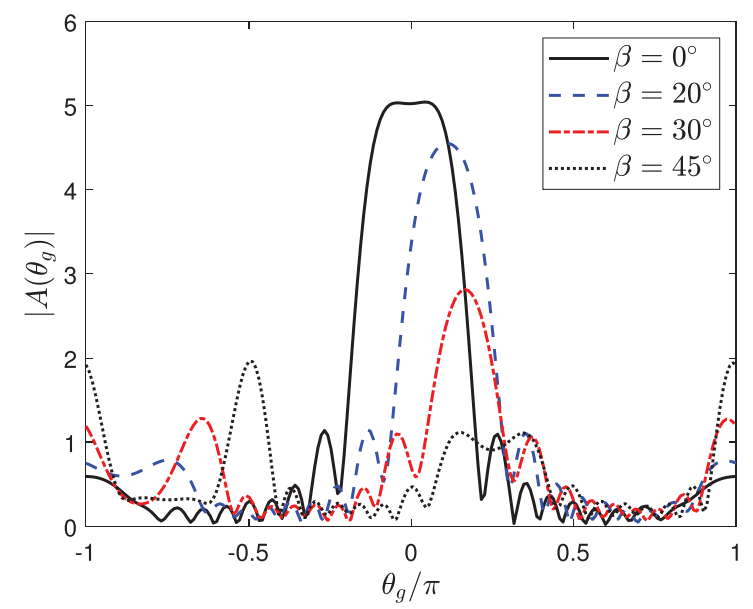

(b)
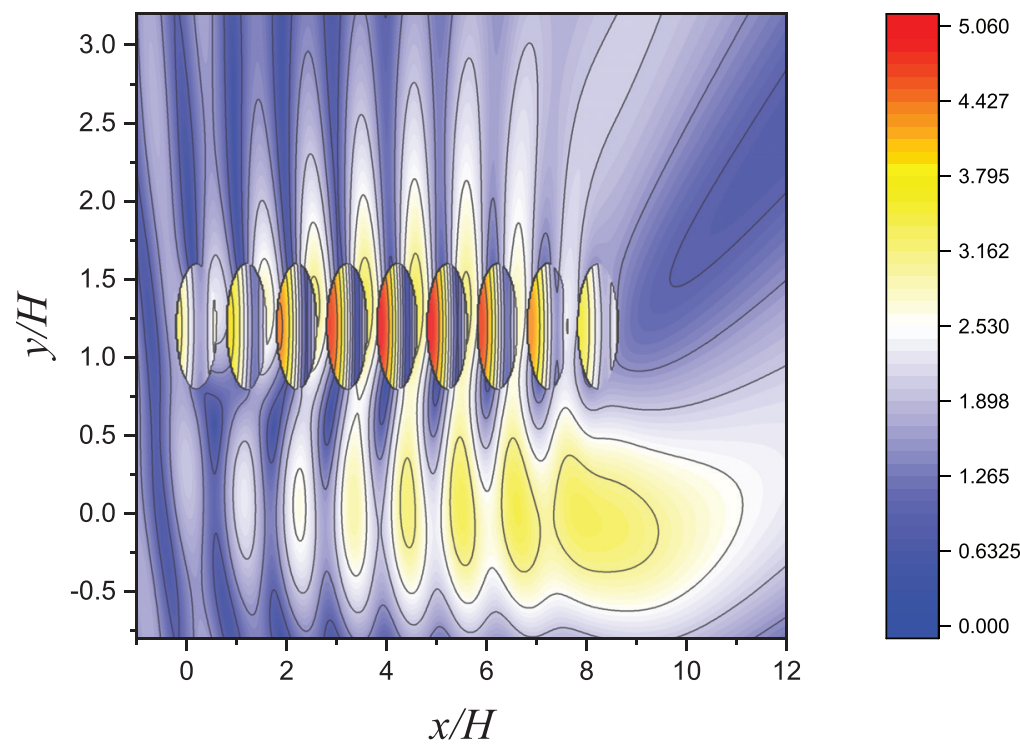

(c)

FIG. 11. Surface wave interaction with an inline arrangement of the multiple fishing showing the (a) scattering coefficient for varying $\left(k_{0} h, \theta_{g}\right)$ at $\beta=30^{\circ}$, (b) scattering coefficient for varying $\theta_{g}$ at various $\beta$ with $k_{0} h=1.25$, and (c) amplitude of surface elevation with $k_{0} h=1.25$ and $\beta=30^{\circ}$. The other parameters are $h / H=0.4$ and $\mu=10^{6} \mathrm{~N} / \mathrm{m}$. 
more energy for larger depth ratio owing to the increased energy dissipation.

\section{Multiple fishing cage}

In Fig. 11, the scattering coefficient and flow distributions around the multiple fishing cage system having an inline arrangement are discussed. In Fig. 11(a), the surface plot depicting the scattering coefficients for varying values of $\left(k_{0} h, \theta_{g}\right)$ is investigated. In the inline arrangement, the incident wave is directed with the phase angle $\beta=30^{\circ}$ toward the system. It is observed that the wave scattering is less for smaller and larger wavenumbers along the direction of the incident wavefield (i.e., $\theta_{g}=30^{\circ}$ ), where most of the incoming waves pass through the system with less scattering. Around $1<k_{0} h<1.5$, the significant portions of waves belonging to this bandwidth range are scattered more, resulting in more reflection from the system. For $k_{0} h=1.25$, the effect of phase angle on the scattering coefficient is plotted in Fig. 11(b). It is noticed that the scattering peaks at an incident wave direction, and it follows the oscillatory pattern along the other direction due to the periodic arrangement of the cage system. However, the wave scattering decreases on either side of the central band due to the inline arrangement, which allows most of the incoming waves through the cage system. Further, the wave scattering decreases with an increasing value of $\beta$ due to the less screening of

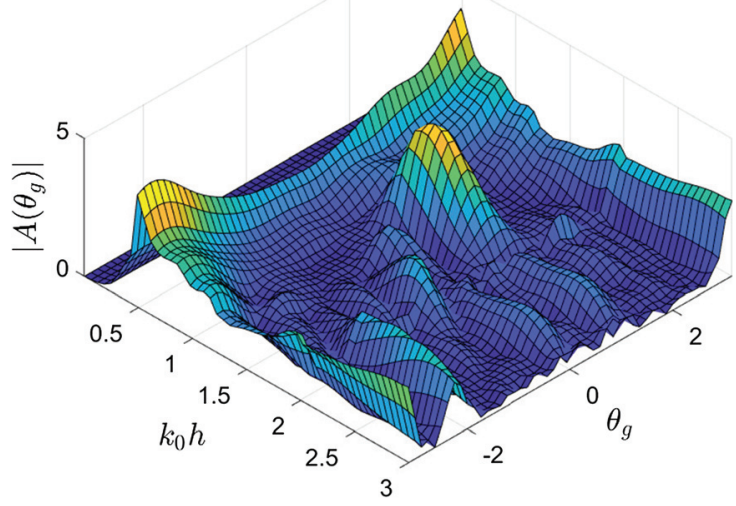

(a)

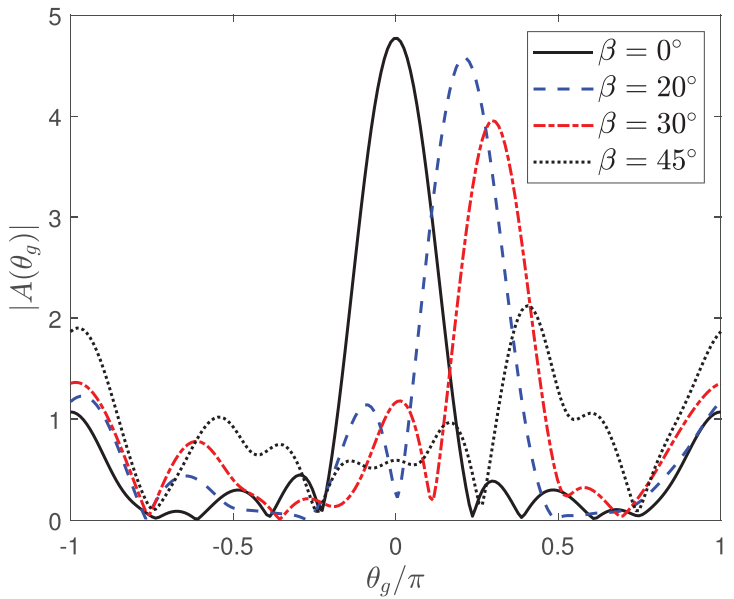

(b)
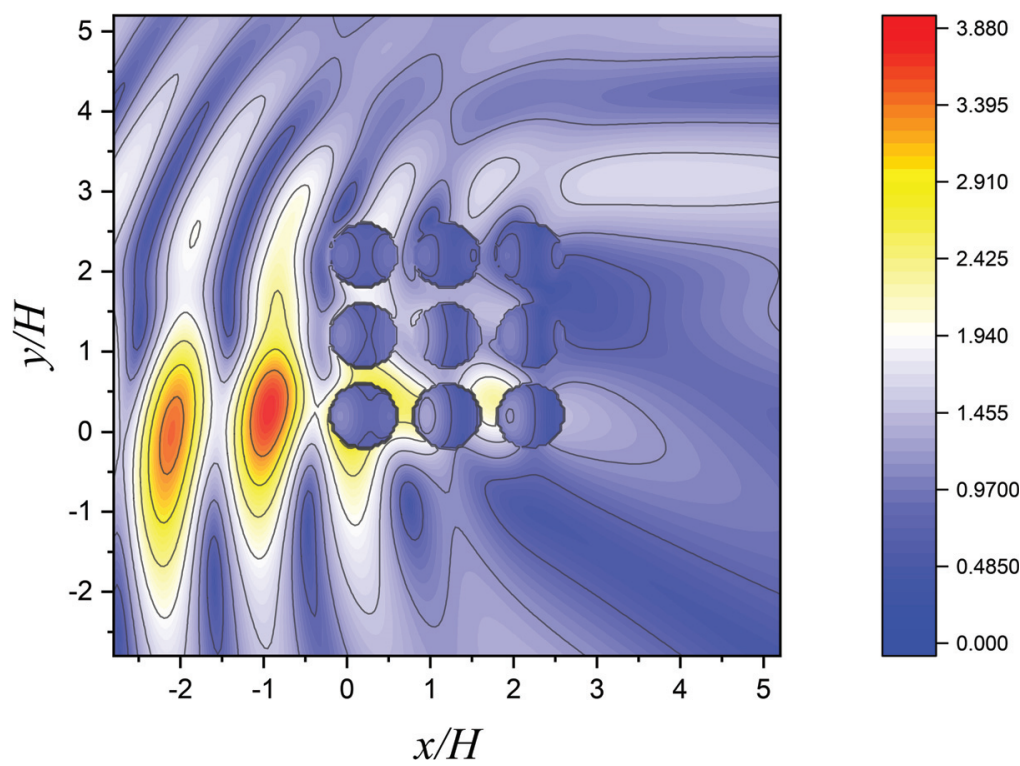

(c)

FIG. 12. Surface wave interaction with a square arrangement of the multiple fishing showing the (a) scattering coefficient for varying $\left(k_{0} h, \theta_{g}\right)$ at $\beta=30^{\circ}$, (b) scattering coefficient for varying $\theta_{g}$ at various $\beta$ with $k_{0} h=1.25$, and (c) amplitude of surface elevation with $k_{0} h=1.25$ and $\beta=30^{\circ}$. The other parameters are $h / H=0.4$ and $\mu=10^{6} \mathrm{~N} / \mathrm{m}$. 
waves by the other cages for a higher phase angle. The corresponding flow distributions around the inline system are demonstrated in Fig. 11(c), where the phase angle of the incident wave is considered as $\beta=30^{\circ}$. In general, the wave amplitude of the surface waves decreases downstream of the cage system due to the wave dissipation by the porous cage system. Furthermore, the wave amplitudes around the cage's circumference increase as a result of scattering between the consequent cages, and accumulated waves inside the cages are dissipated by the vertical porous structure. This reduces the transmission on the lee-side of the cage system.

Figure 12 exhibits the scattering coefficients and flow distributions around the multiple fishing cage system arranged in a square formation. Unlike inline arrangement, there exists a wave scattering for smaller wavenumbers in all directions [Fig. 12(a)]. Then, the scattering coefficients befall and attain a maximum between $1<k_{0} h<1.5$, where more waves are scattered. Furthermore, the wave scattering considerably increases in this arrangement as compared to the

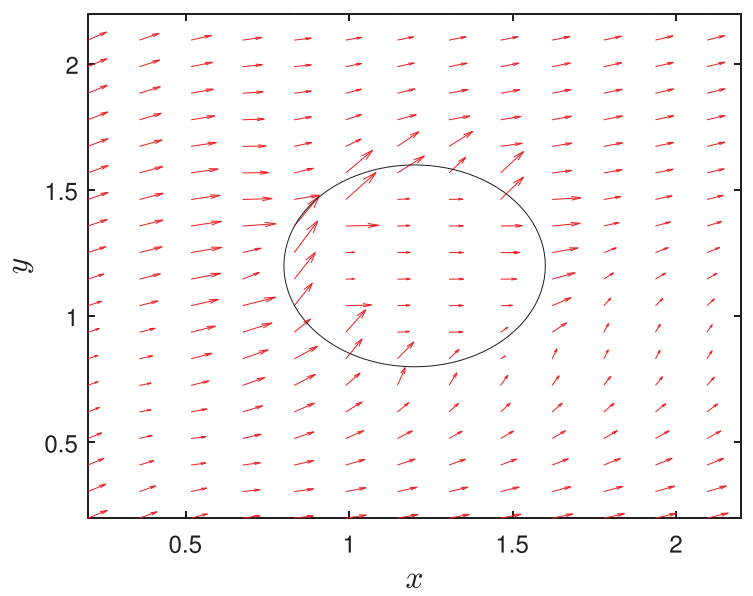

(a) $N=1$

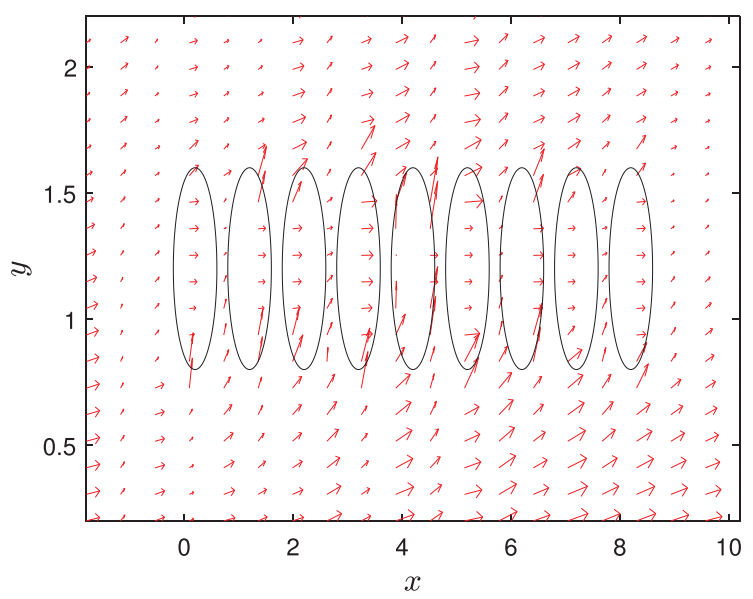

(c) $N=9$ previous arrangement [Fig. 11(a)] due to the wave interaction with the column of cages. By fixing $k_{0} h=1.25$, the response of phase angle on the scattering coefficients is illustrated along a different direction [Fig. 12 (b)]. It is interesting to note that the scattering coefficient increases for a certain optimum angle of $\beta=20^{\circ}$, where most of the waves are scattered. Moreover, the oscillatory patterns are reduced as compared to other arrangements owing to the simultaneous scattering of the incident wave by a column of cylinders. For $\beta>20^{\circ}$, the wave scattering decreases due to the less screening of waves by the cages along the incident wave direction. The flow distributions corresponding to the square arrangement are plotted in Fig. 12(c), where $\beta=30^{\circ}$ is fixed. The constructive interference occurs in between the cages in the first column, and it gradually decreases as the wave passes through the adjacent column of cages. It is observed that the high amplitude waves are damped by an array of fishing cage due to the wave energy dissipation. However, the dissipated wave amplitudes are further trapped inside the system resulting in less wave propagation on the lee-side of

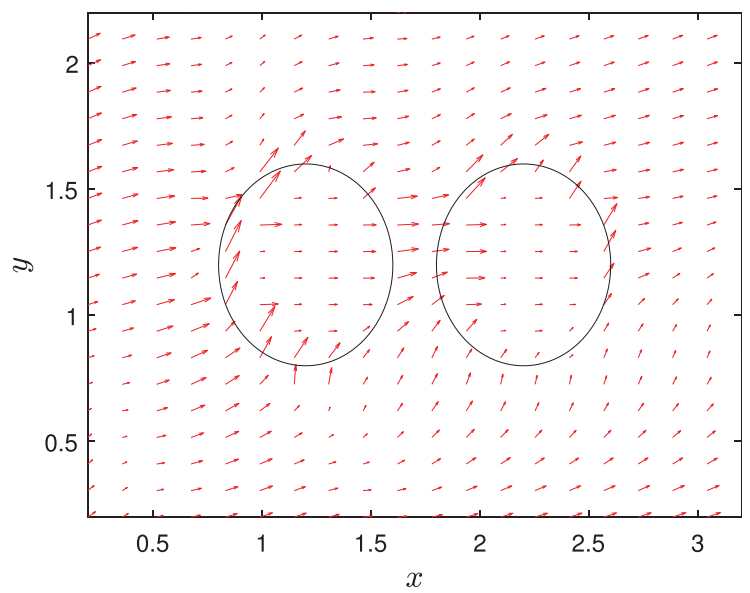

(b) $N=2$

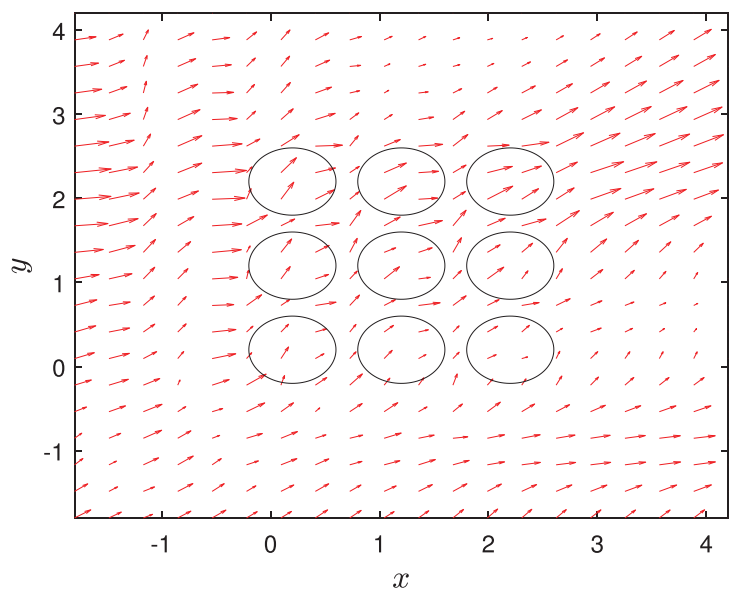

(d) $N=9$

FIG. 13. Flow field around the (a) single fishing cage, (b) dual fishing cage, (c) multiple fishing cage having inline arrangement $(N=9)$, and (d) multiple fishing cage having square arrangement $(N=9)$. The other parameters are $G=3+3 \mathrm{i}, T=0.4, h / H=0.4, \beta=30^{\circ}, k_{0} h=1.25$, and $\mu=10^{6} \mathrm{~N} / \mathrm{m}$. 
the cage system. The damages to the cage system are greatly avoided in such type of arrangement as a consequence of multiple scattering between the cages.

\section{E. Flow field}

In Fig. 13, the flow field around the fishing cage system is plotted for different values of $N$. For $N=1$ [Fig. 13(a)], there is more flow around the circumference of the cage facing the windward side due to the wave interaction. Then, it is passing through the cage, which is due to the wave reflection and dissipation, resulting in less wave transmission in the corresponding lee-ward side. This is true from Fig. 13(a), where the less transmission on the lee-ward side is shown by wellspaced arrows denoting the flow. In the case of a dual fishing cage [Fig. 13(b)], there is an interaction of scattered waves in between the cages resulting in the large accumulation of flow. However, the flow

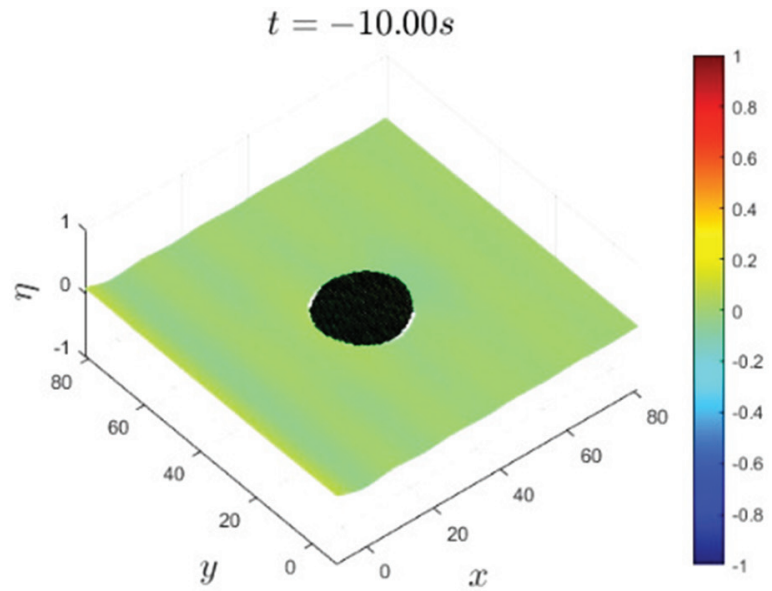

(a)

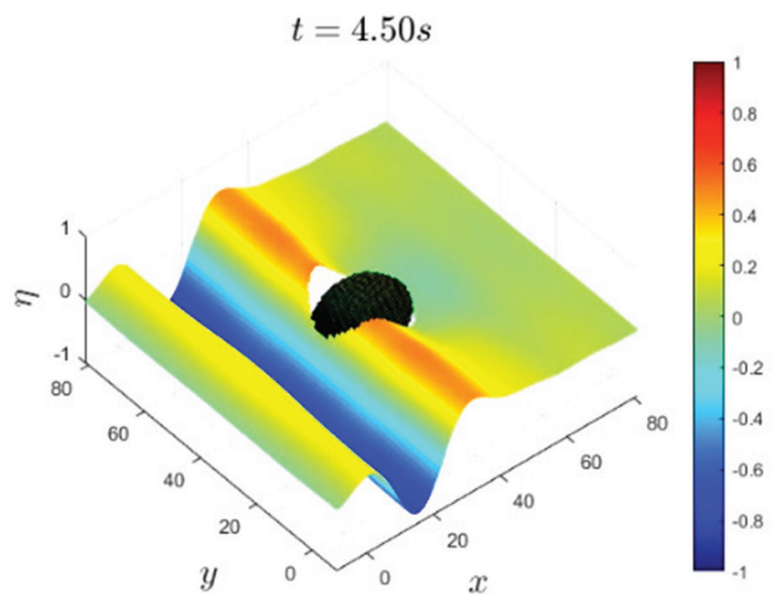

(c) field inside the second cage decreases, resulting in less transmission on the lee-side of the cage system. The flow field through the multiple fishing cages $(N=9)$ arranged in an inline configuration [Fig. 13(c)] shows that the wave passage gradually decreases as it propagates through the cages, which reduces the wave transmission in the lee-side of cages. Moreover, from Fig. 13(d), the wave transmission through the first column of cages decreases for a square configuration. However, there is scattering occurs between the internal cages, and more waves are transmitted on the lee-side of the cage system.

\section{F. Time simulation of solution}

The simulation of a surface elevation $(\eta)$ of the entire domain can be done using the following transformation rule, which can be expressed as:

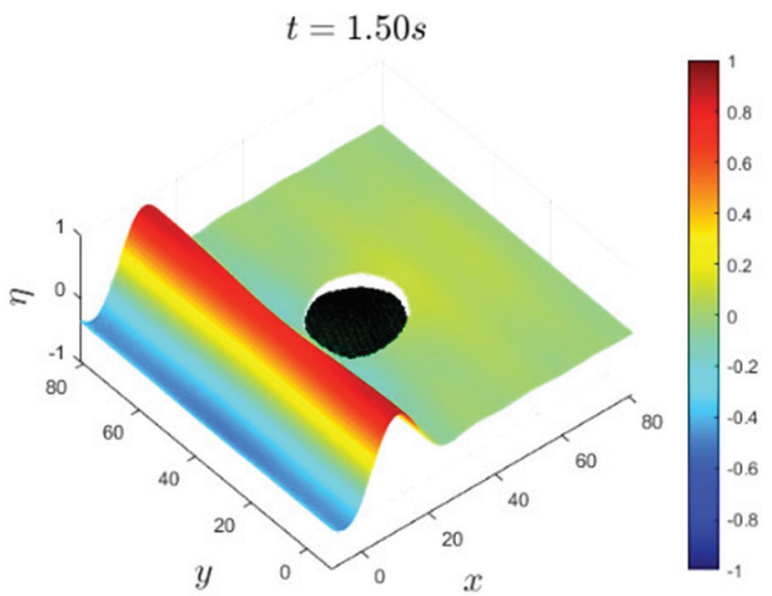

(b)

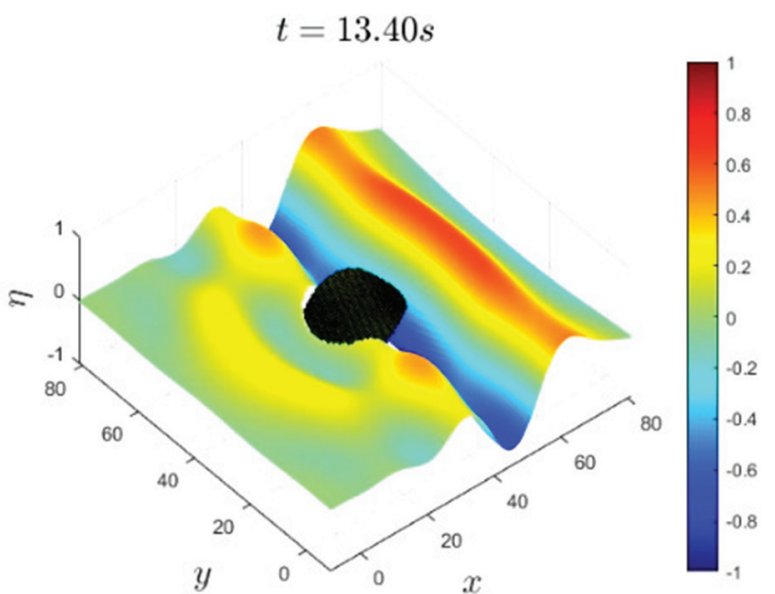

(d)

FIG. 14. Wave scattering by a single fishing cage $(N=1)$ at time (a) $t=-10 \mathrm{~s}$, (b) $t=1.5 \mathrm{~s}$, (c) $t=4.5 \mathrm{~s}$, and (d) $t=13.4 \mathrm{~s}$. The other parameters are $\mathrm{G}=3+3 \mathrm{i}, T=0.4$, $h / H=0.4, \beta=30^{\circ}, k_{0} h=1.25$, and $\mu=10^{6} \mathrm{~N} / \mathrm{m}$. Multimedia view: https://doi.org/10.1063/5.0040662.1 
$\eta(x, y, 0, t)=\Re\left[2 A \sqrt{\pi s} \int_{-\infty}^{\infty} \eta(x, y, 0) \exp \left\{-s\left(\bar{k}-\bar{k}_{c}\right)^{2}-\mathrm{i} \omega t\right\} d \bar{k}\right]$,

where $\Re$ denotes the real part (Meylan, 2021). Further, the parameters such as $s$ and $\bar{k}_{c}$ are known as spreading function and center frequency, respectively. For the purpose of simulation, the values are fixed as $s=2.5$ and $k_{c}=1 / H$.

The time simulation of the wave scattering by the single, double, and triple fishing cage system having the inline arrangement are shown as static Figs. 14(a)-14(c), respectively. The movies corresponding to the static Figs. 14(a)-14(c) are given in the multimedia file. It is noticed that the amplitude of wave interacting with the single fishing cage as in Fig. 14 decreases as it passes through the cage. This happens due to the partial reflection and dissipation from the single fishing cage. In the case of the dual fishing cage system as in Fig. 15, there is a

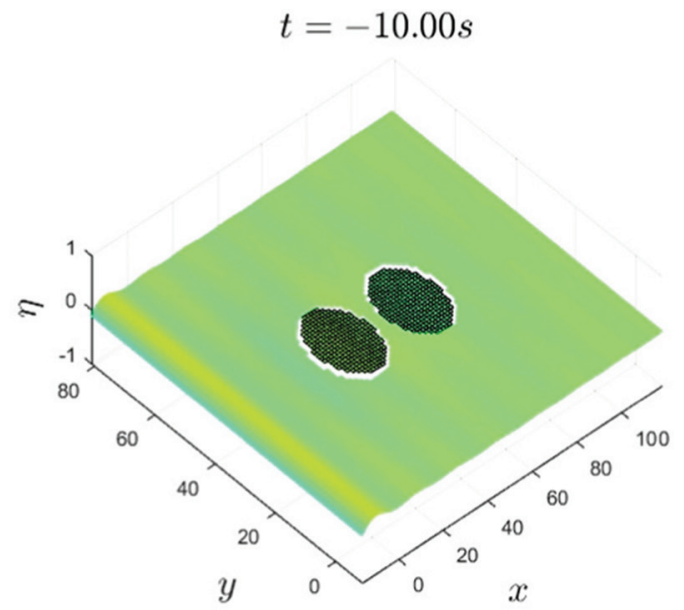

(a)
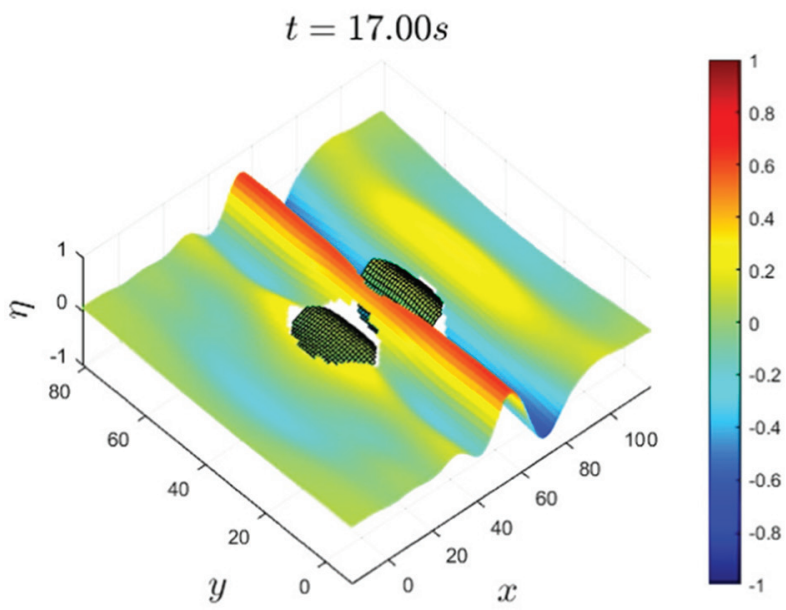

(c) multiple scattering occurs between the cages. This consequently reduces the scattered wave amplitude in both wind-ward and lee-ward sides of the cage system. For the triple cage system as in Fig. 16, at certain wavenumber, the constructive interference occurs resulting in the increased wave amplitude in between the second and third cages in Fig. 16. In general, the wave amplitude in the triple cage system decreases significantly when compared to the single and dual cage system due to the increased rate of energy dissipation and multiple scattering between the cages. Moreover, the wave amplitudes inside the cages are consequently less in Fig. 16 when compared to Figs. 14 and 15. This shows that the fluid circulation becomes smoother inside the cages with an increase in the number of fish cages.

\section{CONCLUSION}

The manuscript deals with the surface wave scattering by the system of multiple fishing cages by employing the linear water wave

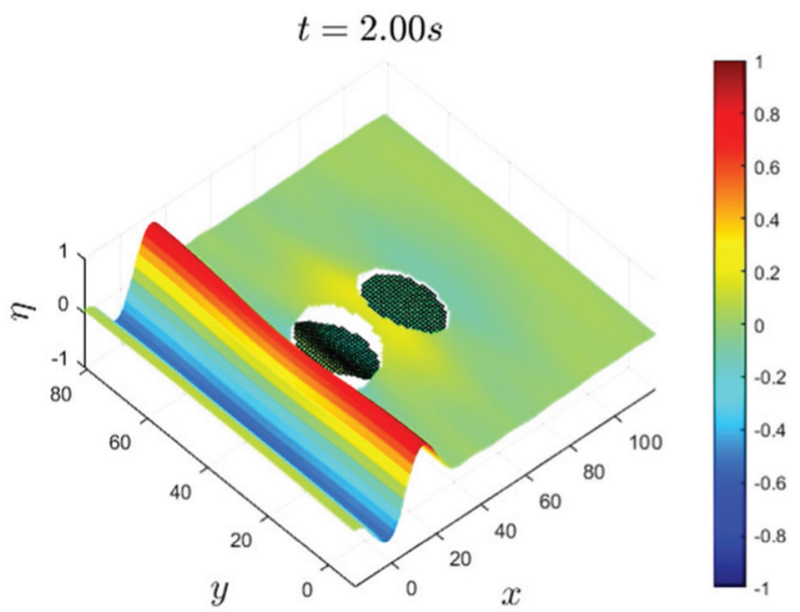

(b)

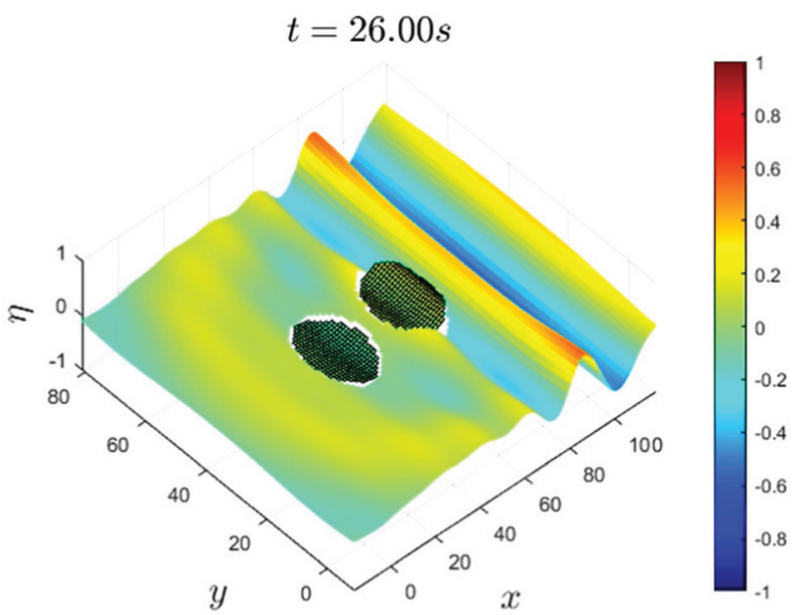

(d)

FIG. 15. Wave scattering by dual fishing cages $(N=2)$ at time (a) $t=-10 \mathrm{~s}$, (b) $t=2 \mathrm{~s}$, (c) $t=17 \mathrm{~s}$, and (d) $t=26 \mathrm{~s}$. The other parameters are $\mathrm{G}=3+3 \mathrm{i}, T=0.4$, $h / H=0.4, \beta=0^{\circ}, k_{0} h=1.25$, and $\mu=10^{6} \mathrm{~N} / \mathrm{m}$. Multimedia view: https://doi.org/10.1063/5.0040662.2 


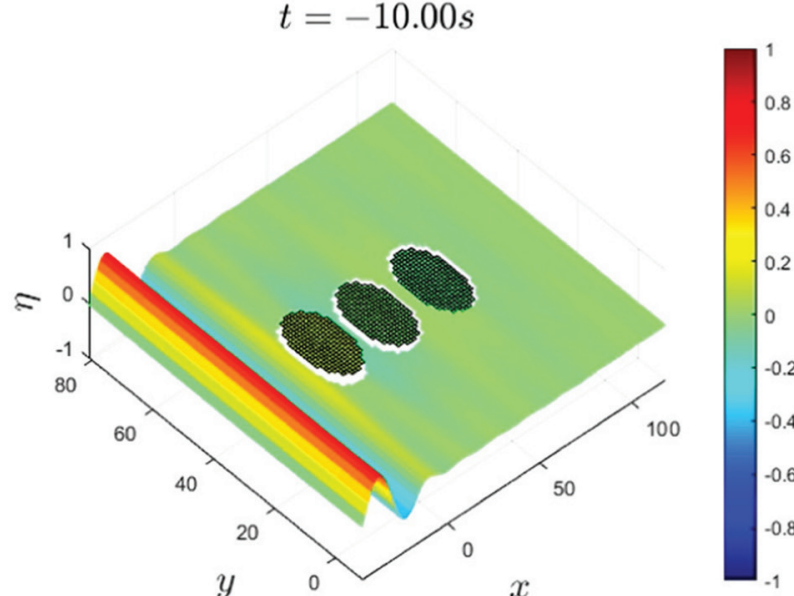

(a)

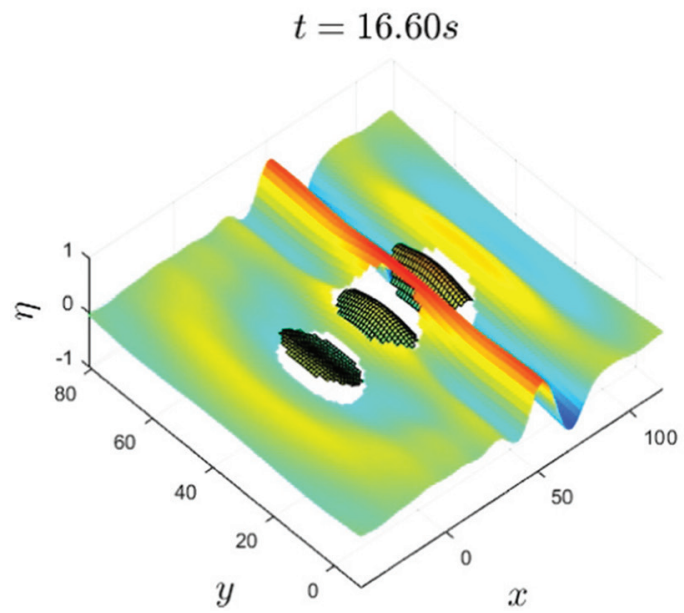

(c)

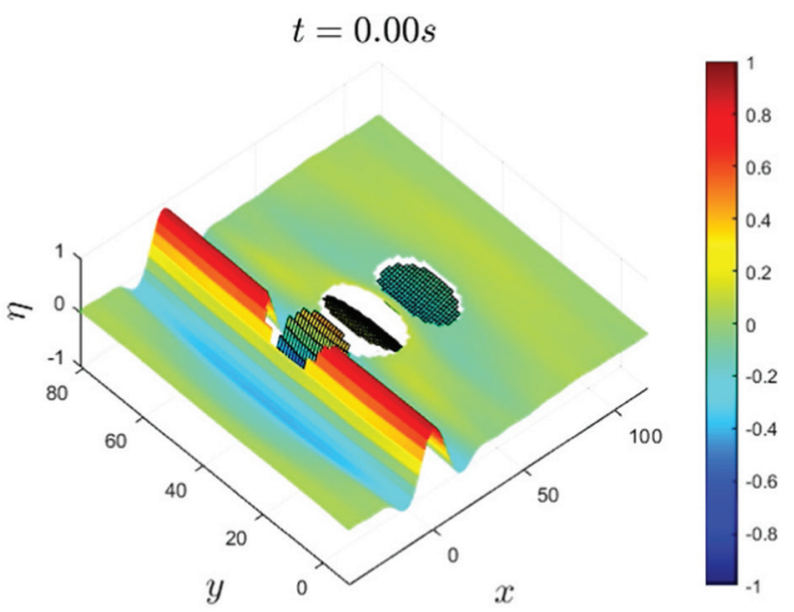

(b)

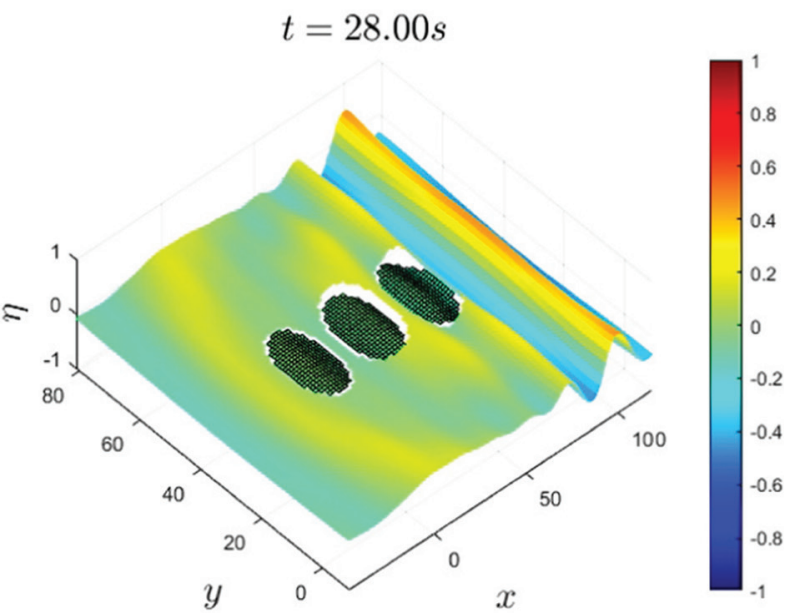

(d)

FIG. 16. Wave scattering by triple fishing cages $(N=3)$ at time (a) $t=-10 \mathrm{~s}$, (b) $t=0 \mathrm{~s}$, (c) $t=16.6 \mathrm{~s}$, and (d) $t=28 \mathrm{~s}$. The other parameters are $G=3+3 \mathrm{i}, T=0.4$, $h / H=0.4, \beta=0^{\circ}, k_{0} h=1.25$, and $\mu=10^{6} \mathrm{~N} / \mathrm{m}$. Multimedia view: https: $/ /$ doi.org $/ 10.1063 / 5.0040662 .3$

theory and the small-amplitude wave response. The general velocity potential in the form of the Fourier-Bessel series is obtained while solving the Laplace equation. Further, the matched eigenfunction expansion and least-square approximation are implemented for solving the system of the linear equation obtained from the interface and edge conditions. The numerical results, such as hydrodynamic wave loads, far-field wave amplitudes, and power dissipation, are investigated for different wave and cage parameters. Moreover, the effect of spatial arrangements on the far-field wave amplitudes and flow distributions are investigated. The following observations are made from the present study:

- The wave amplitude decreases on the lee-side of the cage as a result of wave energy dissipation by the porosity, which consequently increases for the waves with high frequency. The fishing cage connected to the mooring spring of larger spring constant reduces the wave scattering and hydrodynamic wave loads acting on the cage.

- The constructive interference occurs for a small spacing between the cages, which increases the wave amplitude in the confined region between the cages. However, most of the incoming waves inside the cages undergo destructive interference, resulting in the serene environment inside the cages.

- The wave scattering increases with an increasing number of cages and moderate values of tensile force. Further, a fishing cage having a larger height and less tension dissipates more energy as compared to other configurations. Such a cage can act as an efficient power absorber.

- The wave scattered from the multiple fishing cage undergoes more periodic oscillations resulting in the Fabry-Perot effect at a specific direction, where the wave scattering vanishes at the specific directions in far-field. This effect becomes weaker for a 
square arrangement of fishing cages as compared to the inline deployment of the cages.

The occurrence of multiple scattering between the cages, moderate tensile force, and larger porosity reduce the damages caused by the hydrodynamics wave loading due to the incident wave. This leads to the smooth water circulation inside the cages, which can be advantageous for the deep-water fish farming and marine species conservation.

\section{AUTHORS' CONTRIBUTIONS}

S. A. Selvan and R. Gayathri contributed equally to this manuscript.

\section{ACKNOWLEDGMENTS}

H.B. gratefully acknowledges the financial support from SERB, Department of Science and Technology, Government of India through “CRG” project, Award No. CRG/2018/004521.

\section{DATA AVAILABILITY}

The data that support the findings of this study are available within the article, highlighted in the related figure captions and corresponding discussions.

\section{REFERENCES}

Abramowitz, M., and Stegun, I. A., Handbook of Mathematical Functions with Formulas, Graphs, and Mathematical Tables (US Government Printing Office, 1948), Vol. 55

Behera, H., Gayathri, R., and Selvan, S. A., "Wave attenuation by multiple outer porous barriers in the presence of an inner rigid cylinder," J. Waterw. Port Coastal Ocean Eng. 146(1), 04019035 (2020).

Behera, H., and Sahoo, T., "Hydroelastic analysis of gravity wave interaction with submerged horizontal flexible porous plate," J. Fluids Struct. 54, 643-660 (2015).

Behera, H., Selvan, S. A., and Gupta, V. K., "Wave interaction with a floating circular porous elastic plate," in International Conference on Mathematical Modelling and Scientific Computation (Springer, 2018), pp. 85-95.

Chan, A., and Lee, S., "Wave characteristics past a flexible fishnet," Ocean Eng. 28(11), 1517-1529 (2001).

Chwang, A. T., “A porous-wavemaker theory,” J. Fluid Mech. 132, 395-406 (1983).

Duarte, C. M., Holmer, M., Olsen, Y., Soto, D., Marbà, N., Guiu, J., Black, K., and Karakassis, I., "Will the oceans help feed humanity?," Bio Science 59(11), 967-976 (2009).

Fredriksson, D. W., Swift, M. R., Irish, J. D., Tsukrov, I., and Celikkol, B., "Fish cage and mooring system dynamics using physical and numerical models with field measurements," Aquacult. Eng. 27(2), 117-146 (2003).

Golden, C. D., Allison, E. H., Cheung, W. W., Dey, M. M., Halpern, B. S., McCauley, D. J., Smith, M., Vaitla, B., Zeller, D., and Myers, S. S., "Nutrition: Fall in fish catch threatens human health," Nat. News 534(7607), 317 (2016).

Helal, A. M., Attia, A. M., and Mustafa, M. M., "Water conservation and management of fish farm in lake mariout," Life Sci. J. 14(11), 44-51 (2017).

Kagemoto, H., and Yue, D. K., "Interactions among multiple three-dimensional bodies in water waves: An exact algebraic method," J. Fluid Mech. 166, 189-209 (1986).

Karmakar, D., and Soares, C. G., "Oblique scattering of gravity waves by moored floating membrane with changes in bottom topography," Ocean Eng. 54, 87-100 (2012).

Lee, J.-F., and Chen, C.-J., "Wave interaction with hinged flexible breakwater," J. Hydraulic Res. 28(3), 283-297 (1990).
Mandal, S., Datta, N., and Sahoo, T., "Hydroelastic analysis of surface wave interaction with concentric porous and flexible cylinder systems," J. Fluids Struct. 42, 437-455 (2013).

Mandal, S., and Sahoo, T., "Gravity wave interaction with a flexible circular cage system,” Appl. Ocean Res. 58, 37-48 (2016).

McCormick, M. I., Watson, S.-A., and Munday, P. L., "Ocean acidification reverses competition for space as habitats degrade,” Sci. Rep. 3(1), 1-6 (2013).

Meylan, M. et al., "A flexible vertical sheet in waves," in The Fourth International Offshore and Polar Engineering Conference (International Society of Offshore and Polar Engineers, 1994).

Meylan, M. H., "Time-dependent motion of a floating circular elastic plate," Fluids 6(1), 29 (2021).

Meylan, M. H., Bennetts, L. G., and Peter, M. A., "Water-wave scattering and energy dissipation by a floating porous elastic plate in three dimensions," Wave Motion 70, 240-250 (2017).

Park, M.-S., Jeong, Y.-J., and You, Y.-J., "Water wave interaction by dual cylindrical cylinders with partial porous area,” Int. J. Eng. Technol. 9(2), 97 (2017).

Park, M.-S., You, Y.-J., and Jeong, Y.-J., "Numerical analysis of an offshore platform with partial porous cylinders due to wave excitation forces and seismic forces," in IEEE Proceedings of 2012 Oceans (2012), pp. 1-9.

Sarkar, A., and Bora, S. N., "Hydrodynamic forces due to water wave interaction with a bottom-mounted surface-piercing compound porous cylinder," Ocean Eng. 171, 59-70 (2019a).

Sarkar, A., and Bora, S. N., "Water wave diffraction by a surface-piercing floating compound porous cylinder in finite depth," Geophys. Astrophys. Fluid Dyn. 113(4), 348-376 (2019b).

Selvan, S. A., and Behera, H., "Wave energy dissipation by a floating circular flexible porous membrane in single and two-layer fluids," Ocean Eng. 206, 107374 (2020).

Selvan, S. A., Behera, H., and Sahoo, T., "Reduction of hydroelastic response of a flexible floating structure by an annular flexible permeable membrane," J. Eng. Math. 118(1), 73-99 (2019).

Su, W., Zhan, J.-M., and Huang, H., "Analysis of a porous and flexible cylinder in waves," China Ocean Eng. 29(3), 357-368 (2015).

Williams, A., Geiger, P., and McDougal, W., "Flexible floating breakwater," J. Waterw. Port Coastal Ocean Eng. 117(5), 429-450 (1991).

Williams, A., Li, W., and Wang, K.-H., "Water wave interaction with a floating porous cylinder," Ocean Eng. 27(1), 1-28 (2000).

Yu, X., and Chwang, A. T., "Wave-induced oscillation in harbor with porous breakwaters," J. Waterw. Port Coastal Ocean Eng. 120(2), 125-144 (1994).

Zhai, Z., Huang, H., Ye, W., Yang, L., and Liu, S., "Hydrodynamic interactions between cnoidal waves and a concentric cylindrical structure with arc-shaped outer cylinder," Ocean Eng. 209, 107448 (2020).

Zhang, L., Ulgiati, S., Yang, Z., and Chen, B., "Emergy evaluation and economic analysis of three wetland fish farming systems in nansi lake area," China. J. Environ. Manage. 92(3), 683-694 (2011).

Zhao, F., Bao, W., Kinoshita, T., and Itakura, H., "Theoretical and experimental study on a porous cylinder floating in waves," J. Offshore Mech. Arct. Eng. 133(1), 011301 (2011).

Zhao, Y.-P., Bi, C.-W., Chen, C.-P., Li, Y.-C., and Dong, G.-H., "Experimental study on flow velocity and mooring loads for multiple net cages in steady current," Aquacult. Eng. 67, 24-31 (2015).

Zhao, Y.-P., Bi, C.-W., Dong, G.-H., Gui, F.-K., Cui, Y., Guan, C.-T., and Xu, T.J., "Numerical simulation of the flow around fishing plane nets using the porous media model," Ocean Eng. 62, 25-37 (2013).

Zheng, S., Meylan, M., Greaves, D., and Iglesias, G., "Water-wave interaction with submerged porous elastic disks," Phys. Fluids 32(4), 047106 (2020a).

Zheng, S., Meylan, M. H., Fan, L., Greaves, D., and Iglesias, G., "Wave scattering by a floating porous elastic plate of arbitrary shape: A semi-analytical study," J. Fluids Struct. 92, 102827 (2020b).

Zheng, S., Meylan, M. H., Zhu, G., Greaves, D., and Iglesias, G., "Hydroelastic interaction between water waves and an array of circular floating porous elastic plates," J. Fluid Mech. 900, A20 (2020c). 


\section{University Library}

\section{- M M N E R VA A gateway to Melbourne's research publications}

Minerva Access is the Institutional Repository of The University of Melbourne

Author/s:

Selvan, SA;Gayathri, R;Behera, H;Meylan, MH

Title:

Surface wave scattering by multiple flexible fishing cage system

Date:

2021-03-01

Citation:

Selvan, S. A., Gayathri, R., Behera, H. \& Meylan, M. H. (2021). Surface wave scattering by multiple flexible fishing cage system. PHYSICS OF FLUIDS, 33 (3), https:// doi.org/10.1063/5.0040662.

Persistent Link:

http://hdl.handle.net/11343/297473 